\title{
Abundances in metal-rich stars ${ }^{\star}$
}

\section{Detailed abundance analysis of $47 \mathrm{G}$ and $\mathrm{K}$ dwarf stars with $[\mathrm{Me} / \mathrm{H}]>0.10 \mathrm{dex}$}

\author{
S. Feltzing ${ }^{1,2}$ and B. Gustafsson ${ }^{2}$ \\ 1 Royal Greenwich Observatory, Madingley Road, Cambridge CB3 0EZ, UK \\ 2 Astronomiska observatoriet, Box 515, S-751 20 Uppsala, Sweden
}

Received June 25; accepted September 26, 1997

\begin{abstract}
We have derived elemental abundances of $\mathrm{O}$, $\mathrm{Na}, \mathrm{Mg}, \mathrm{Al}, \mathrm{Si}, \mathrm{Ca}, \mathrm{Ti}, \mathrm{Cr}, \mathrm{Mn}, \mathrm{Fe}, \mathrm{Co}, \mathrm{Ni}$ as well as for a number of $s$-elements for $47 \mathrm{G}$ and $\mathrm{K}$ dwarf, with $[\mathrm{Me} / \mathrm{H}]>0.1 \mathrm{dex}$. The selection of stars was based on their kinematics as well as on their $u v b y-\beta$ photometry. One sample of stars on rather eccentric orbits traces the chemical evolution interior to the solar orbit and another, on circular orbits, the evolution around the solar orbit. A few Extreme Population I stars were included in the latter sample.
\end{abstract}

The stars have -0.1 dex $<[\mathrm{Fe} / \mathrm{H}]<0.42$ dex. The spectroscopic $[\mathrm{Fe} / \mathrm{H}]$ correlate well with the $[\mathrm{Me} / \mathrm{H}]$ derived from $u v b y-\beta$ photometry. We find that the elemental abundances of $\mathrm{Mg}, \mathrm{Al}, \mathrm{Si}, \mathrm{Ca}, \mathrm{Ti}, \mathrm{Cr}$ and $\mathrm{Ni}$ all follow $[\mathrm{Fe} / \mathrm{H}]$. Our data put further constraints on models of galactic chemical evolution, in particular of $\mathrm{Cr}, \mathrm{Mn}$ and Co which have not previously been studied for dwarf stars with $[\mathrm{Me} / \mathrm{H}]>0.1$ dex. The increase in $[\mathrm{Na} / \mathrm{Fe}]$ and $[\mathrm{Al} / \mathrm{Fe}]$ as a function of $[\mathrm{Fe} / \mathrm{H}]$ found previously by Edvardsson et al. (1993a) has been confirmed for $[\mathrm{Na} / \mathrm{Fe}]$. This upturning relation, and the scatter around it, are shown not to be due to a mixture of populations with different mean distances to the galactic centre. We do not confirm the same trend for aluminium, which is somewhat surprising since both these elements are thought to be produced in the same environments in the pre-supernova stars. Nor have we been able to trace any tendency for relative abundances of $\mathrm{O}, \mathrm{Si}$, and Ti relative to Fe to vary with the stellar velocities, i.e. the stars present mean distance to the galactic centre. These results imply that there is no significant difference in the chemical evolution of the different stellar populations for stars with $[\mathrm{Me} / \mathrm{H}]>0.1$ dex. We find that $[\mathrm{O} / \mathrm{Fe}]$ continue to decline with increasing $[\mathrm{Fe} / \mathrm{H}]$ and that oxygen and europium correlate well.

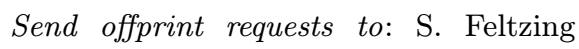

sofia@ast.cam.ac.uk

* Based on observations at the McDonald Observatory.
However $[\mathrm{Si} / \mathrm{Fe}]$ and $[\mathrm{Ca} / \mathrm{Fe}]$ seem to stay constant. A real ("cosmic") scatter in $[\mathrm{Ti} / \mathrm{Fe}]$ at given $[\mathrm{Fe} / \mathrm{H}]$ is suggested as well as a decreasing abundance of the $s$-elements relative to iron for the most metal-rich dwarf stars. We discuss our results in the context of recent models of galactic chemical evolution.

In our sample we have included a few very metal rich stars, sometimes called SMR (super metal rich) stars. We find these stars to be among the most iron-rich in our sample but far from as metal-rich as indicated by their photometric metallicities. SMR stars on highly eccentric orbits, alleged to trace the evolution of the chemical evolution in the galactic Bulge, have previously been found overabundant in $\mathrm{O}, \mathrm{Mg}$ and $\mathrm{Si}$. We have included three such stars from the study by Barbuy \& Grenon (1990). We find them to be less metal rich and the other elemental abundances remain puzzling.

Detailed spectroscopic abundance analyses of K dwarf stars are rare. Our study includes $5 \mathrm{~K}$ dwarf stars and has revealed what appears to be a striking example of overionization. The overionization is especially prominent for $\mathrm{Ca}, \mathrm{Cr}$ and $\mathrm{Fe}$. The origin of this apparent overionization is not clear and we discuss different explanations in some detail.

Key words: Galaxy: abundances — evolution — stars: abundances - late-type — fundamental parameters

\section{Introduction}

The time that $\mathrm{F}$ and $\mathrm{G}$ dwarf stars spend on the mainsequence span a range from $10^{9}$ to several times $10^{10}$ years. This means that such stars may be used as tracers of the chemical and dynamical evolution of the Galaxy; in fact, a combination of chemical and kinematical data is a very powerful tool for studying the galactic chemical evolution, 
cf. e.g. Edvardsson et al. (1993a) and Wyse \& Gilmore (1995).

Spectroscopic abundance analysis of stars have now become accurate enough to admit determinations of rather small ( $\lesssim 0.10$ dex) relative abundance differences in differential studies of stellar samples of large size. These developments make it possible to explore the galactic chemical evolution in considerable detail.

Edvardsson et al. (1993a) analysed $189 \mathrm{~F}$ and $\mathrm{G}$ dwarf stars, with -1.1 dex $<[\mathrm{Fe} / \mathrm{H}]<0.25$ dex. Accurate abundances were determined for a number of key elements, $\mathrm{O}$, $\mathrm{Na}, \mathrm{Mg}, \mathrm{Al}, \mathrm{Si}, \mathrm{Ca}, \mathrm{Ti}, \mathrm{Fe}$ and $\mathrm{Ni}$, as well as a number of $s$-process elements. The abundance results combined with accurate velocity data enabled a detailed study of the chemical evolution of kinematically distinct populations. Extensions of this study were made by Tomkin et al. (1995) and Woolf et al. (1995) who measured carbon and europium abundances, respectively, for about half of the stars in the Edvardsson et al. (1993a) sample.

The study by Edvardsson et al. (1993a) raised a number of new questions concerning the most metal-rich stars in the galactic disk, not the least concerning the buildup of sodium, magnesium and aluminium. Sodium, aluminium and, possibly, magnesium relative iron vs. $[\mathrm{Fe} / \mathrm{H}]$ showed an increase for $[\mathrm{Fe} / \mathrm{H}]>0.0$ dex (cf. Edvardsson et al. 1993a Figs. 15a-l). Are these "upturns" real? Large star-to-star scatter was also encountered for the abundance of certain elements: magnesium, aluminium and titanium, relative to iron at a given $[\mathrm{Fe} / \mathrm{H}]$. Could this scatter be reduced by using more/better abundance criteria or is the scatter intrinsic to the stellar population? One suggestion was that "upturns" and scatter could be due to a mixing of populations with different ages and with different mean distances from the galactic centre, e.g. a mixture of old metal-rich stars, more concentrated to the centre and young Extreme Population I stars on solar like orbits. We have therefore studied a sample of 47 metal-rich dwarf stars, with photometric metallicities $[\mathrm{Me} / \mathrm{H}]>0.10 \mathrm{dex}$, chosen to represent different mean perigalactic distances and presumably different ages.

The paper is organized as follows: in Sects. 2 and 3, we describe the selection criteria of the stellar sample, the observations and reductions, Sect. 4 contains a description of the analysis while the errors are discussed in detail in Sect. 5. Abundance results and their interpretation in terms of models of galactic chemical evolution are presented in Sect. 6 and, finally, Sect. 7 contains a summary and discussion.

\section{Selection of stars}

The stars have been selected to include both stars from the old metal-rich disk population, as well as more recently formed Extreme Population I dwarf stars. The sample of stars was confined photometrically by $0.38<b-y<0$ m 63 and with $\delta c_{1}$ and $\Delta m_{1}$ such that with the calibrations of Edvardsson et al. (1993a) and Olsen (1984), solartype dwarfs and subgiants are singled out with $4500 \mathrm{~K}$ $\leq T_{\text {eff }} \leq 6000 \mathrm{~K}, 4.0 \leq \log g \leq 4.6$ and $[\mathrm{Me} / \mathrm{H}]>0.10 \mathrm{dex}$. Magnitude and metallicity data were taken from the catalogues by Olsen (1983, 1993, 1994 and priv. comm.). These were combined with accurate velocity data (UVW) calculated by Olsen from available proper motions and unpublished CORAVEL radial velocities by Andersen, Mayor \& Nordström, in the selection of programme stars.

From the catalogue we seleted $42 \mathrm{G}$ and $\mathrm{K}$ dwarf stars with $[\mathrm{Me} / \mathrm{H}]>0.10$ for our study. 13 of these stars have $V_{\mathrm{LSR}}<-50 \mathrm{~km} \mathrm{~s}^{-1}$ and/or $Q_{\mathrm{LSR}}>60 \mathrm{~km} \mathrm{~s}^{-1}$, where $V_{\mathrm{LSR}}$ is the stellar space velocity component in the direction of the rotation of the Galaxy relative to the local standard of rest (LSR) and $Q_{\mathrm{LSR}}$ is the total space velocity relative to LSR. These stars were selected to represent the chemical evolution galactic regions different from those represented by the solar orbit, in particular located more closely to the galactic centre. As shown in Edvardsson et al. (1993b), Fig. 1, $V_{\mathrm{LSR}}<-50 \mathrm{~km} \mathrm{~s}^{-1}$ singles out stars with mean perigalactica less than $7 \mathrm{kpc}$. The 17 stars with $Q_{\mathrm{LSR}}<30 \mathrm{~km} \mathrm{~s}^{-1}$ represent the chemical evolution in the gas close to the solar orbit.

Since we also wished to explore the oxygen abundances in metal-rich disk dwarf stars on highly eccentric orbits we added 3 additional stars from the sample of stars for which Barbuy \& Grenon (1990) found abnormally high [O/Fe] ratios (HD 37986, HD 77338, HD 87007). Moreover, we included 2 stars from Schuster \& Nissen (1988) (HD 175518 and HD 182572).

The total final sample contains 47 stars and has 3 in common with Edvardsson et al. (1993a): HD 30562, HD 67228 and HD 144585.

\section{Observations and reductions}

\subsection{Observations}

The observations were carried out with the $2.7 \mathrm{~m}$ telescope of the McDonald Observatory, University of Texas, during two observing runs, March 1-9, 1994 and April 28 May 2, 1994, using the 2d-coudé cross dispersion echelle spectrometer (Tull et al. 1995).

At the time of the observations the new $2 \mathrm{k} \times 2 \mathrm{k}$ chip, which covers most of the visual spectrum of the observed star in one exposure, was not yet available at the spectrometer. The CCD-chip used has $800 \times 864$ pixels and covers approximately 18 echelle orders of length $25-$ $35 \AA$. Settings of the detector for the observations were selected to enable reliable analysis of the spectral lines from the elements oxygen, sodium, magnesium and aluminium, silicon, calcium, titanium, as well as chromium, iron, nickel, yttrium and europium. Many sources were utilized to make the selection of lines optimized for the current project, the most important being Edvardsson et al. (1993a), Morell (1994), Mäckle et al. (1975), Ruland et al. 
(1981). The Arcturus Atlas by Griffin (1968) and the Solar Spectrum Atlas by Delbouille et al. (1973), were carefully inspected, in order to avoid blends and define suitable continuum regions. Altogether 3 different settings of the CCD in the focal plane of the spectrometer camera were used.

The nominal spectral resolution (slit width $0.25 \mu \mathrm{m}$ ) of $\sim 100000$, was verified by observations of a thoriumargon lamp and by measuring telluric lines. This relatively high resolution was judged to be important in view of the relatively crowded spectra, in particular for the cooler stars.

\subsection{Reductions of stellar spectra}

The reductions were carried out with standard IRAF $^{1}$ packages for reduction and extraction of echelle spectra. The procedure adopted was: all raw frames were first corrected for read-out noise and bias, the flat fields added together, all object frames were trimmed, the new flat field frames were normalized, the stellar frames were divided by the normalized flat field, corrected for scattered light and the one-dimensional stellar spectra extracted. Finally, the spectrum of the comparison lamp, a thorium-argon lamp, was reduced, extracted and the lines in it identified. The comparison spectra were used to carry out the wavelength calibration of the stellar spectra. A Legendre polynomial was fitted to each stellar spectrum to define the continuum, using the CONTINUUM task in IRAF.

The results of this procedure for defining the continuum were inspected visually and judged to give good continua for all the lines used in the abundance analysis; however, for the strong Ca I line at $6162 \AA$, used for checking surface gravities, this definition of the continuum was not satisfactory for all stars. Instead, a blaze function was constructed for each night by fitting a Legendre polynomial to the spectrum of a B star observed during the night. The stellar spectra were then divided by this blaze function. Then a low order Legendre polynomial was fitted to the stellar spectrum to rectify it. The change in continuum was negligible for half of the stars, as compared with the result of using CONTINUUM; this also includes half of the $\mathrm{K}$ dwarf stars.

Our spectra are generally of high quality, reaching $\mathrm{S} / \mathrm{N}$ of $\sim 200$ in most cases. Some problems with fringes in the red were encountered. The measurements of spectral line equivalent widths were done using the SPLOT task in IRAF. Karin Eriksson is gratefully thanked for carrying out almost half of the measurements. Care was exercised to guarantee that the measurements agreed well between the two operators.

\footnotetext{
$\overline{1}$ IRAF is distributed by National Optical Astronomy Observatories, operated by the Association of Universities for Research in Astronomy, Inc., under contract with the National Science Foundation, U.S.A.
}

\subsection{Solar observations and reductions}

The solar observations were performed by illuminating the slit of the spectrograph by sky light each afternoon. The solar spectra have $\sim 2$ times higher $\mathrm{S} / \mathrm{N}$ ratios than the stellar spectra. The reductions and measurements of the solar spectra were made in the same way as for the stellar spectra.

The solar observations were used to determine "astrophysical" $\log g f$-values. For lines, denoted by $\mathrm{K}$ in Table 2 , shifted by the difference in radial velocities between the stars and the Sun outside our solar spectral recordings, but judged to be of interest for the analyses, solar equivalent widths were instead obtained from the solar spectrum atlas by Kurucz et al. (1984).

\section{Analysis}

We have performed a standard Local Thermodynamic Equilibrium (LTE) analysis, strictly differential with respect to the Sun, to derive chemical abundances from the measured equivalent widths.

\subsection{Model atmospheres}

We used the MARCS program, first described by Gustafsson et al. (1975), to generate the model atmospheres. Since then, the program has been further developed in various ways and updated in order to handle the line blanketing of millions of absorption lines more accurately, Asplund et al. (1997). The following assumptions enter into the calculation of the models: the atmosphere is assumed to be plane-parallel and in hydrostatic equilibrium, the total flux (including mixing-length convection) is constant, the source function is described by the Planck function at the local temperature with a scattering term, the populations of different excitation levels and ionization stages are governed by LTE. Since the analysis is differential relative to the Sun we have also used a solar model atmosphere calculated with the same program as the stellar models, in spite of the fact that the empirically derived Holweger-Müller model better reproduces the solar observed limb darkening; see Blackwell et al. (1995) for a discussion of this.

\subsection{Fundamental parameters of model atmospheres}

The effective temperature and surface gravity for each star were derived from $u v b y-\beta$ photometry from Olsen (1983, 1993, 1994 and priv. comm.). We have used an extension of the calibration in Edvardsson et al. (1993a) and the calibration in Olsen (1984). The calibration by Edvardsson et al. (1993a) is valid for the parameter space $2.58<\beta<2.72$ and $0.04<\left(\delta c_{1}+0.5 \cdot \delta m_{1}\right)<0.16$. This translates roughly to $5600 \mathrm{~K}<T_{\text {eff }}<7000 \mathrm{~K}, 3.8<$ $\log g<4.5$ and metallicity $-1 \mathrm{dex}<[\mathrm{Me} / \mathrm{H}]<+0.3$ dex. The Edvardsson et al. (1993a) calibration was preferred 
Table 1. Stellar parameters of the observed stars. The fifth column gives the spectral classification according to Olsen (1983, 1993, 1994 and priv. comm.), except where indicated 1) from Eggen (1960), and 2) spectral class from Hoffleit \& Jascheck (1982); the sixth and seventh columns give effective temperature and surface gravity as derived from photometry; the eight and ninth give surface gravities as derived from spectra, wings of Ca I, and parallaxes, respectively; the tenth and eleventh give iron abundances as determined from our spectral analysis and metallicities as determined from photometry; the following four columns contain the space velocity data for the stars, in $\mathrm{km} \mathrm{s}^{-1}$ relative to the LSR, from Olsen (priv. comm.). In the last column the stellar space velocity is given

\begin{tabular}{|c|c|c|c|c|c|c|c|c|c|c|c|c|c|c|c|}
\hline HD & $\mathrm{HR}$ & Name & $V$ & Spec.class & $T_{\text {eff }}$ & $\log g_{\text {phot }}$ & $\log g_{\text {spec }}$ & $\log g_{\pi}$ & {$[\mathrm{Fe} / \mathrm{H}]$} & {$[M / H]$} & $\xi_{\mathrm{t}}$ & $U$ & $V$ & $W$ & $Q$ \\
\hline 30562 & 1536 & & 5.77 & F8V & 5876 & 4.00 & 4.00 & 3.9 & 0.19 & 0.14 & 1.4 & 40.8 & -70.9 & -10.7 & 82.5 \\
\hline 36130 & & & 7.76 & G0M & 5986 & 4.34 & 4.40 & 4.0 & 0.15 & 0.15 & 1.1 & -14.3 & -52.5 & -43.7 & 69.8 \\
\hline 37088 & & & 8.51 & G0 & 5856 & 4.26 & 4.35 & & 0.10 & 0.31 & 1.8 & -17.4 & -12.4 & -43.0 & 48.0 \\
\hline 37216 & & & 7.84 & G5M & 5527 & 4.47 & 4.90 & & -0.02 & 0.17 & 1.1 & 9.8 & 1.0 & -4.3 & 10.7 \\
\hline 49178 & & & 8.07 & G0E & 5683 & 4.37 & 4.45 & & 0.01 & 0.06 & 1.2 & 32.3 & 10.3 & -10.5 & 35.5 \\
\hline 54322 & & & 8.40 & G5 & 5894 & 4.50 & 4.55 & & 0.15 & 0.33 & 0.9 & -20.2 & -0.7 & 7.1 & 21.4 \\
\hline 55693 & & & 7.18 & G5M & 5845 & 4.15 & 4.40 & & 0.26 & 0.24 & 1.3 & 33.9 & 3.3 & -5.0 & 34.4 \\
\hline 67228 & 3176 & & 5.30 & GIVB & 5831 & 4.14 & 3.90 & 3.6 & 0.16 & 0.22 & 1.4 & -41.9 & 16.6 & -9.2 & 46.0 \\
\hline 68988 & & & 8.20 & G0 & 5956 & 4.03 & 4.25 & & 0.37 & & 1.4 & -94.1 & -8.8 & 11.8 & 95.2 \\
\hline 69582 & & & 7.56 & G5 & 5652 & 4.34 & 4.74 & & 0.08 & 0.06 & 1.2 & -20.0 & 11.5 & 10.5 & 25.3 \\
\hline 69830 & 3259 & & 5.96 & G7.5V & 5484 & 4.30 & 4.95 & 4.5 & -0.03 & 0.10 & 1.1 & -41.3 & -51.7 & -4.4 & 66.3 \\
\hline 71479 & & & 7.18 & G0 & 6036 & 4.18 & 4.48 & & 0.25 & 0.32 & 1.5 & 34.8 & -40.4 & -9.1 & 54.1 \\
\hline $72946 B$ & 3396 & & 7.20 & G5V & 5911 & 4.40 & 5.00 & & 0.24 & 0.40 & 1.3 & 15.0 & -18.0 & -3.4 & 23.7 \\
\hline 75782 & & & 7.08 & G0 & 5930 & 3.87 & 3.77 & & 0.18 & 0.11 & 1.5 & 12.3 & -23.0 & -9.1 & 27.7 \\
\hline 76780 & & & 7.64 & G5M & 5869 & 4.30 & 4.80 & & 0.21 & 0.30 & 1.3 & 22.3 & -6.7 & 10.1 & 25.3 \\
\hline $80607 \mathrm{~A}$ & & & 9.15 & G5R & 5457 & 4.28 & & & 0.27 & 0.30 & 1.1 & -16.9 & 15.0 & 19.3 & 29.7 \\
\hline 87646 & & & 8.07 & G0 & 5961 & 4.06 & 4.41 & & 0.30 & 0.39 & 1.4 & 21.9 & -11.4 & 2.6 & 24.8 \\
\hline 91204 & & & 7.82 & G0 & 5864 & 4.05 & 4.00 & & 0.17 & 0.24 & 1.4 & -19.6 & 9.7 & 3.6 & 22.2 \\
\hline 94835 & G 058 & $8-030$ & 9.11 & $\mathrm{~K} 0 / \mathrm{G} 0$ & 5896 & 4.06 & & & 0.13 & 0.04 & 1.4 & & -65.1 & & \\
\hline 101242 & & & 7.61 & G5 & 5790 & 4.28 & 4.68 & & 0.07 & 0.19 & 1.3 & 45.2 & -50.0 & -2.6 & 67.4 \\
\hline 106156 & & & 7.92 & K0 & 5437 & 4.27 & 4.77 & & 0.13 & 0.20 & 1.1 & -61.9 & -13.0 & -11.3 & 64.3 \\
\hline 110010 & & & 7.01 & G0 & 5965 & 4.08 & 4.58 & 4.7 & 0.35 & 0.34 & 1.4 & 9.0 & -14.1 & -6.6 & 17.9 \\
\hline 117243 & & & 8.35 & G0/G5III ${ }^{1}$ & 5902 & 4.01 & 4.36 & & 0.24 & 0.33 & 1.0 & 13.2 & -59.9 & 4.6 & 61.5 \\
\hline 125968 & & & 7.77 & G0/G5IV-V ${ }^{1}$ & 5868 & 4.12 & 4.32 & 4.7 & 0.15 & 0.25 & 1.4 & 22.6 & -89.6 & -27.7 & 96.5 \\
\hline 126511 & & & 8.37 & G5 & 5472 & 4.28 & 4.70 & & 0.06 & 0.10 & 1.3 & -14.5 & -47.6 & -9.9 & 50.7 \\
\hline 128987 & & & 7.24 & G5 & 5588 & 4.35 & 5.00 & & 0.05 & 0.10 & 0.9 & 14.7 & 2.9 & -7.1 & 16.6 \\
\hline 130087 & & & 7.52 & F5 & 6023 & 4.01 & 4.41 & & 0.25 & 0.25 & 1.5 & 13.0 & -16.6 & 4.9 & 21.7 \\
\hline 134474 & & & 8.88 & G5 & 5375 & 4.46 & 5.06 & & 0.16 & 0.33 & 1.0 & 7.7 & 22.8 & -27.1 & 36.2 \\
\hline 134987 & 5657 & $23 \mathrm{Lib}$ & 6.47 & $\mathrm{G} 4 \mathrm{~V}$ & 5833 & 4.11 & 4.31 & 4.0 & 0.36 & 0.58 & 1.3 & 10.3 & -24.9 & 26.9 & 38.1 \\
\hline 137510 & 5740 & & 6.27 & G0IV-V & 5929 & 3.91 & 3.91 & & 0.25 & 0.28 & 1.5 & -0.2 & 2.0 & 7.6 & 7.9 \\
\hline 144585 & 5996 & & 6.31 & G4IV-V & 5831 & 4.03 & 4.38 & & 0.27 & 0.26 & 1.4 & 23.6 & -17.5 & 33.6 & 44.6 \\
\hline 171999A & & & 8.33 & G5 & 5249 & 4.32 & 4.65 & 4.0 & 0.40 & 0.06 & 0.9 & 7.6 & -61.3 & -3.3 & 61.9 \\
\hline 175518 & & & & $\mathrm{~K} 0 \mathrm{IV}-\mathrm{V}^{1}$ & 5713 & 3.93 & 4.73 & 4.3 & 0.32 & 0.67 & 1.5 & 14.6 & -99.8 & 12.6 & 101.7 \\
\hline $178911 \mathrm{~A}$ & 7272 & & 6.73 & G5R & 5910 & 4.24 & 4.44 & 4.0 & 0.06 & 0.35 & & 35.1 & -8.8 & 6.0 & 36.7 \\
\hline 180890 & & & 8.35 & G5 & 5530 & 4.23 & 4.53 & & 0.14 & 0.09 & 1.5 & 16.9 & -50.0 & -9.4 & 53.6 \\
\hline 182572 & 7373 & $31 \mathrm{Aql}$ & 6.36 & $\mathrm{G} \mathrm{IV}^{2}$ & 5739 & 3.83 & 4.43 & 4.1 & 0.42 & 0.50 & 1.4 & -1.0 & & & 123.2 \\
\hline 183263 & & & 7.87 & G5/G2IV & 5837 & 4.05 & 4.40 & & 0.15 & 0.22 & 1.5 & 18.6 & -32.2 & 8.6 & 38.2 \\
\hline 186427 & 7504 & 16 Cyg B & 6.23 & G3V & 5773 & 4.17 & 4.42 & & 0.06 & 0.21 & 1.3 & -27.9 & -17.9 & 5.8 & 33.6 \\
\hline 187055 & & & 9.00 & G5 & 5298 & 4.56 & 4.96 & 3.9 & 0.16 & 0.25 & 0.9 & 83.0 & 5.3 & -12.7 & 84.1 \\
\hline \multicolumn{16}{|c|}{ K dwarf stars } \\
\hline 32147 & 1614 & & 6.21 & K3V & 4625 & 4.57 & 4.55 & 4.4 & 0.28 & 0.17 & 1.0 & -11.5 & -36.1 & -3.7 & 38.0 \\
\hline $61606 \mathrm{~A}$ & & & 7.17 & $\mathrm{~K} 2 \mathrm{~V}$ & 4833 & 4.55 & 4.85 & 4.6 & -0.08 & 0.11 & 1.0 & -34.9 & 9.4 & -1.0 & 36.1 \\
\hline 103932 & & & 6.95 & $\mathrm{~K} 5 \mathrm{~V}$ & 4510 & 4.58 & 4.85 & 4.6 & 0.16 & 0.21 & 1.0 & 9.9 & -59.9 & 0.4 & 60.7 \\
\hline $131977 \mathrm{~A}$ & 5568 & & 5.72 & $\mathrm{~K} 4 \mathrm{~V}$ & 4585 & 4.58 & 4.70 & 4.6 & 0.04 & 0.18 & 1.0 & -57.2 & -9.5 & -26.1 & 63.6 \\
\hline 136834 & & & 8.26 & K0 & 4765 & 4.56 & 4.47 & 4.5 & 0.19 & 0.23 & 1.0 & 9.6 & -44.3 & -8.7 & 46.2 \\
\hline \multicolumn{16}{|c|}{ Stars in common with Barbuy \& Grenon (1990) } \\
\hline $37986^{3}$ & & & 7.37 & G5/K0IV & 5455 & 4.50 & 4.40 & 4.3 & 0.27 & 0.47 & 1.0 & & & & \\
\hline $77338^{3}$ & & & 8.63 & K0IV & 5290 & 4.50 & 4.90 & & 0.22 & 0.45 & 1.0 & & & & \\
\hline $87007^{3}$ & & & 8.81 & $\mathrm{~K} 2$ & 5300 & 4.50 & 4.70 & & 0.27 & 0.43 & 1.0 & $40.3^{4}$ & $-42.5^{4}$ & $-14.2^{4}$ & \\
\hline
\end{tabular}

${ }^{3}$ All stellar parameters from Barbuy \& Grenon (1990).

${ }^{4}$ Barbuy, private communication. 
for all stars to which it is applicable. For the three stars previously studied by Barbuy \& Grenon (1990) uvby $-\beta$ photometry is not available, and we used the stellar parameters cited by them.

\subsection{Surface gravity}

Surface gravities were derived from the $c_{1}$ index, which primarily measures the Balmer jump and is sensitive to the surface gravity in solar type dwarf stars. In addition to surface gravities estimated from photometry we have also used the wings of the strong calcium line at $6162 \AA$ (see Blackwell \& Willis 1977 and Edvardsson 1988), to derive surface gravities. The results are given in Table 1 . For these results we are indebted to Matthias Palmer and Mikael Nilsson, who carried out these laborious determinations. Edvardsson et al. (1993a) estimated the error in surface gravity determined from their calibration of the $c_{1}$ index to 0.2 dex. Olsen (1984) quotes a similar accuracy for his calibration. For the stars with trigonometric parallaxes in van Altena et al. (1991) we have also estimated surface gravities from parallaxes. This exercise was meant to be a consistency check on the photometric and spectroscopic determinations of the surface gravities. Assuming that the stars have masses of $0.8 M_{\odot}$ and interpolating the bolometric corrections in the table given in Allen (1973) we derived, from the parallaxes, the surface gravities (see e.g. Gustafsson et al. 1974) shown in Fig. 1 and given in Table $1^{2}$.

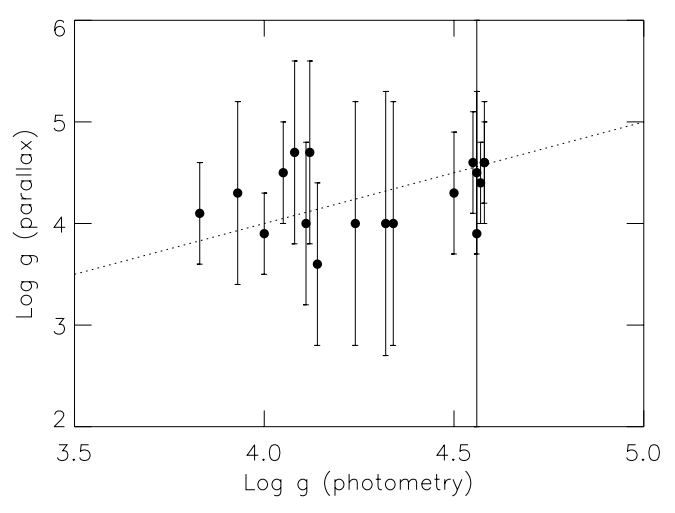

Fig. 1. Surface gravities derived from parallaxes vs. surface gravities derived from photometry. The one-to-one relation is indicated by the dotted line. The error bars reflect the errors in the parallaxes. Note the different scales on $x-$ and $y$-axis

The agreement between surface gravities determined with the different methods is in general good. We note,

\footnotetext{
2 With the release of the Hipparcos parallaxes this can now be addressed in greater detail and for most of our stars. The results will be published elsewhere.
}

however, that there is in the mean an offset in the surface gravities determined from the wings of the strong Ca I line as compared to the surface gravities determined from photometry, see Table 1 , of about +0.27 dex. The reason for this offset is not clear but, a corresponding uncertainty in surface gravity is of minor significance for the abundance results, see Table 3. A tendency for the trigonometric $\log g$ determiantions to agree closer with the photometric than with the spectroscopic ones may also be traced. It is interesting to note that the $\mathrm{K}$ dwarf stars in our study have $\log g$ derived from parallaxes that are in good agreement with those determined from photometry.

On the basis of these comparisons we found no reason to change the surface gravities to be used in the abundance analysis but kept those determined from photometry.

\subsection{Microturbulence parameters}

We have determined microturbulence parameters, $\xi_{\mathrm{t}}$, from both the CaI lines and the Fe I lines for 12 of the stars with enough calcium and iron lines measured. For the determination from the CaI lines, abundances from the individual lines were derived with $\xi_{\mathrm{t}}$ ranging from 0.10 to $1.90 \mathrm{~km} \mathrm{~s}^{-1}$. The microturbulence parameter for each star was then determined as the $\xi_{\mathrm{t}}$ value which gave the smallest abundance scatter (the inflexion point, see e.g. Smith 1981). The microturbulence parameters were also determined by plotting the iron abundance versus the reduced equivalent widths, $\log \left(W_{\lambda} / \lambda\right)$, derived for each line. If the correct microturbulence parameter is used the slope of a fit to the data points should be zero. Both methods agreed well for these 12 stars.

Using the values obtained we derived a relation between effective temperature, surface gravity and the microturbulence parameter, which was then used to determine microturbulence parameters for the rest of the stars: $\xi_{\mathrm{t}}=4.510^{-4} T_{\text {eff }}-0.31 \cdot \log g$ and predicts, with $T_{\text {eff }}$ and $\log g$ adopted, $\xi_{\mathrm{t}}$ values to an accuracy of $\pm 0.1 \mathrm{~km} \mathrm{~s}^{-1}$ for the 12 stars. This relation is valid for $3.93<\log g<4.50$ and $5530 \mathrm{~K}<T_{\text {eff }}<6036 \mathrm{~K}$. For a few stars just outside the validity range we extrapolated the relation to calculate approximative microturbulence parameters. This procedure was checked to yield consistent abundances for individual iron lines of different strengths. For five stars a somewhat lower microturbulence parameter was preferred (HD 36130, HD 134474, HD 171999, HD 182572, HD 187055). Our relation for microturbulence parameters yield slightly higher values than the relation presented by Edvardsson et al. (1993a).

For the $\mathrm{K}$ dwarf stars none of the described methods seemed to yield definite values for the microturbulence parameter. A microturbulence parameter of $1.0 \mathrm{~km} \mathrm{~s}^{-1}$ was adopted for these stars. 
Table 2. Column 1: Wavelength as quoted in Moore et al. (1966). Column 2: Excitation energy of the lower level involved in the transition. Column 3: Astrophysically derived oscillator strengths (based on the solar equivalent widths). Column 4: Correction factor to Unsöld's damping constant. Column 5: Radiative damping constant. Column 6: Equivalent width as measured in the Sun. If nothing else is indicated in the 7th column observations of the daylight sky with the same spectrograph were used. Column 7: Lines used to check the excitation equilibrium are denoted by $\mathrm{t}$ and lines for which the solar equivalent width was measured from the Kurucz et al. (1984) Solar Flux Atlas by a K, see Sect. 3.3. Cr II and Fe II lines excluded from the analysis of the K dwarfs stars due to possible blends are denoted by +, see Sect. 5.8. For each element the solar logarithmic abundance is given according to in Anders \& Grevesse (1989), except for iron for which the value 7.51 was used, see Holweger et al. (1991) and Biémont et al. (1991)

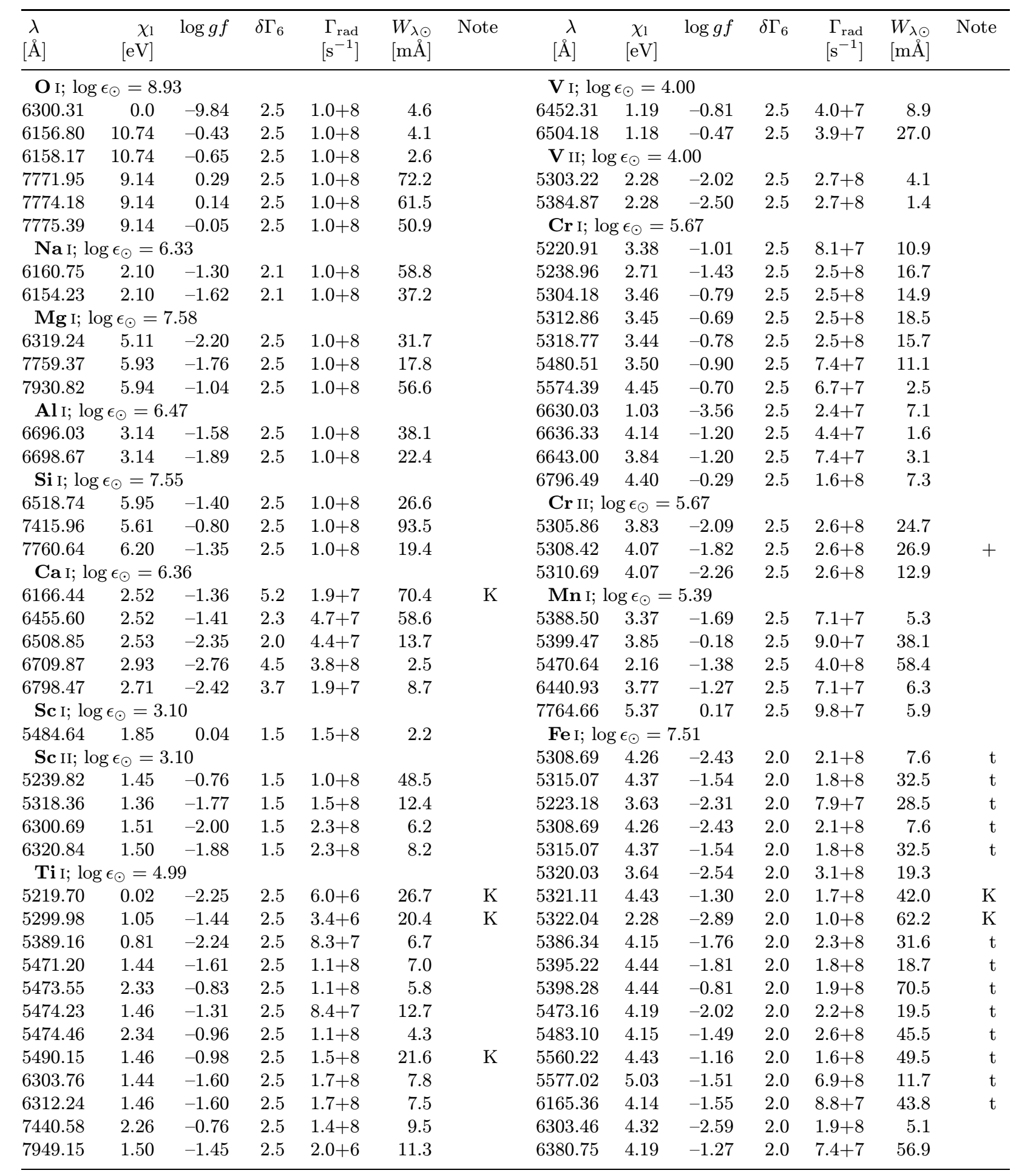


Table 2. continued

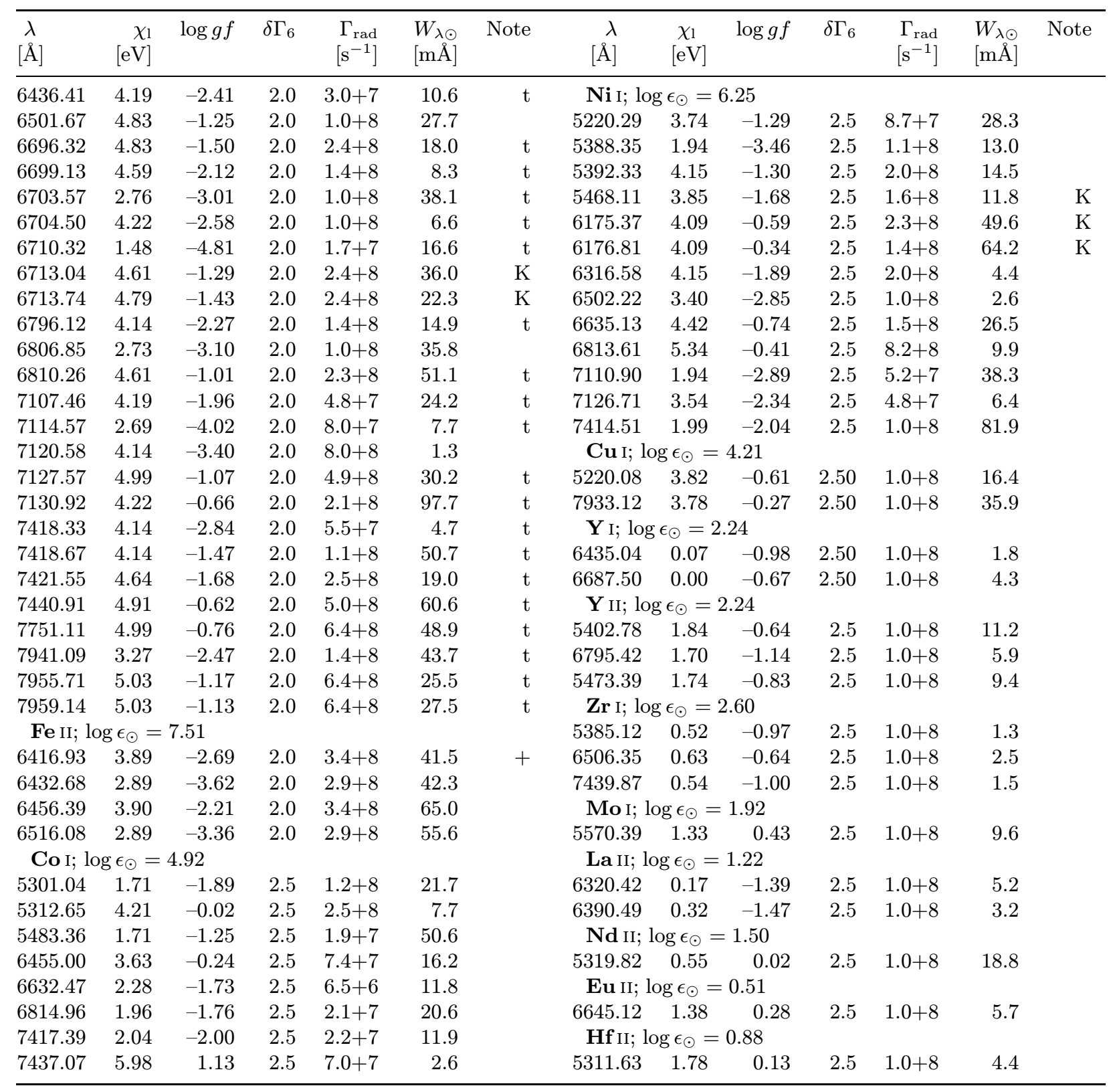

\subsection{Atomic line data}

The oscillator strengths were determined by requiring that the abundances calculated for the solar model $\left(T_{\text {eff }}=\right.$ $\left.5780 \mathrm{~K}, \log g=4.44,[\mathrm{Me} / \mathrm{H}]=0.00, \xi_{\mathrm{t}}=1.00\right)$ should reproduce the observed equivalent widths of the solar spectrum. The resulting $\log g f$ values are given in Table 2 .

Different line broadening mechanisms, van der Waals damping, radiation damping, thermal Doppler broadening and microturbulence were considered in the calculations of equivalent widths and abundances. Enhancement factors for the van der Waals damping were compiled from the literature. For iron lines values from Hannaford et al. (1992) and Holweger et al. (1991) were used, for calcium values from Smith (1981) and references therein, for sodium values from Holweger (1971) and for scandium values from Neuforge (1992). For the remaining lines a correction factor of 2.5 was adopted to the classical Unsöld value, according to Mäckle et al. (1975). For the (unimportant) radiation damping parameter values from Kurucz (1989) were adopted for lines from calcium through nickel. 
Table 3. Effects on abundance estimates for a number of elements from changes in fundamental parameters of the model atmosphere. The resulting abundances are shown for 3 stars: HD $72946\left(T_{\text {eff }} / \log g /[\mathrm{Fe} / \mathrm{H}]\right)=(5911 / 4.4 / 0.24), \mathrm{HD} 103932$ (4510/4.58/0.16), and HD 110010 (5965/4.08/0.35). The first line for each star gives $[\mathrm{X} / \mathrm{H}], \mathrm{X}$ being the ion indicated in the header, derived using a model atmosphere constructed with the stellar parameters adopted in this study. The following lines contain the differences when effective temperature or surface gravity has been changed as indicated in the first column. Note that the changes in parameters are different, and larger, for HD 103932

\begin{tabular}{|c|c|c|c|c|c|c|c|c|c|c|c|c|c|}
\hline ID $/ \Delta$ & {$[\mathrm{O} \mathrm{I}]$} & $\mathrm{NaI}$ & $\mathrm{Mg} \mathrm{I}$ & $\mathrm{Al} \mathrm{I}$ & S I & $\mathrm{CaI}$ & Ti I & Cr I & Cr II & $\mathrm{Fe} I$ & Fe II & $\mathrm{Ni} I$ & $\mathrm{Eu} \mathrm{I}$ \\
\hline HD 72946 & 0.16 & 0.22 & 0.24 & 0.23 & 0.13 & 0.22 & 0.38 & 0.24 & 0.09 & 0.24 & 0.00 & 0.18 & 0.09 \\
\hline$+100 \mathrm{~K}$ & +0.01 & +0.04 & +0.04 & +0.06 & +0.01 & +0.06 & +0.08 & +0.06 & -0.03 & +0.05 & +0.01 & +0.05 & 0.00 \\
\hline$+0.2 \mathrm{dex}$ & +0.09 & -0.03 & -0.01 & -0.01 & -0.01 & -0.02 & 0.00 & 0.00 & +0.08 & -0.01 & +0.12 & +0.01 & +0.08 \\
\hline HD 103932 & 0.28 & & 0.30 & 0.14 & 0.27 & -0.12 & +0.03 & 0.01 & 0.44 & 0.14 & 0.57 & 0.41 & 0.32 \\
\hline$+200 \mathrm{~K}$ & +0.05 & & -0.08 & +0.12 & -0.18 & +0.18 & +0.24 & +0.12 & -0.18 & -0.04 & -0.32 & -0.05 & -0.01 \\
\hline$+0.4 \mathrm{dex}$ & +0.16 & & +0.02 & -0.04 & +0.10 & -0.04 & -0.02 & +0.02 & +0.19 & +0.07 & +0.21 & +0.09 & +0.15 \\
\hline HD 110010 & -0.04 & 0.53 & 0.48 & 0.40 & 0.51 & 0.35 & 0.29 & 0.30 & 0.22 & 0.35 & 0.33 & 0.31 & 0.16 \\
\hline$+100 \mathrm{~K}$ & +0.01 & +0.05 & +0.04 & +0.04 & +0.01 & +0.06 & +0.08 & +0.06 & +0.03 & +0.06 & -0.05 & +0.05 & 0.00 \\
\hline+0.2 dex & +0.09 & -0.02 & -0.01 & -0.02 & -0.01 & -0.01 & 0.00 & 0.00 & +0.08 & -0.01 & -0.03 & 0.00 & +0.09 \\
\hline
\end{tabular}

\section{Errors in resulting abundances}

\subsection{Measurement of equivalent widths and fitting of continua}

If we assume that the true continuum level is not wildly different from the fitted continuum the error in measured equivalent width can at the most be as large, in difficult cases, as $2 \mathrm{~m} \AA$ for weak lines, i.e. $\sim 20 \%$, and $4-5 \mathrm{~m} \AA$ for stronger lines, i.e. $\sim 10 \%$. This translates to typically 0.08 dex in the resulting abundance derived from a line of strength $10 \mathrm{~m} \AA$ and 0.04 dex for a line of strength $50 \mathrm{~m} \AA$. Apart from the effects of the continuum errors and blends, the error in derived abundances due to errors in the measurement of the equivalent width of a line is negligible $(<0.01 \mathrm{dex})$. In general, the lines and continua are, due to the high $\mathrm{S} / \mathrm{N}$ and high resolution, easy to fit and the errors given above should be regarded as upper limits.

\subsection{Oscillator strengths}

The oscillator strengths, derived from the observed solar spectrum, can be affected by misidentification, by undetected blends and by errors in continuum fitting and measurements of equivalent widths in the solar spectrum. As in the stellar spectra, location of the continuum is a much larger source of error than the actual measurement of a line. (Note, however, that the solar spectra have higher $\mathrm{S} / \mathrm{N}$, usually $\sim 400$, and thus, identification of the continuum becomes easier as well as identification of lines. The Sun is also more metal-poor than the programme stars, which makes identification of the continuum in the Sun easier.) Using the results in Sect. 5.1 we find that errors in $\log g f$-values may be as large as $0.08 \mathrm{dex}$, but a more characteristic number is 0.04 dex.

Since we perform a purely differential analysis errors due to misplaced continua, neglected blends, etc. should partly cancel in the first approximation and not affect the resulting differential abundances very much, as long as we study stars similar to the Sun.

\subsection{Blends}

In the selection of lines we have carefully avoided all lines that could be subject to blending with nearby lines as given in Moore et al. (1966). For ions with several lines measured we have also looked for lines which produces abnormally high abundances as compared with the majority of the lines. This led us to exclude three Fe II lines from our final analyses: 6383.71, 6383.45, and 6627.32 A.

\subsection{Fundamental parameters of the model atmospheres}

Edvardsson et al. (1993a) estimate the error in the effective temperature due to errors in $b-y$ to range from $-50 \mathrm{~K}$ to $+100 \mathrm{~K}$ and the corresponding error in $\log g$ to be \pm 0.2 dex.

The effects of such errors in $T_{\text {eff }}$ and $\log g$ are exemplified in Table 3. As expected, abundances derived from ions are most sensitive to changes in surface gravity while abundances derived from atoms are most sensitive to changes in effective temperature. In general, errors in derived abundances are smaller than 0.10 dex for atoms when varying the effective temperature by $\pm 100 \mathrm{~K}$ and less than 0.02 dex when the surface gravity is varied by \pm 0.20 dex; they are smaller than 0.02 dex for ions when the effective temperature is varied and less than $0.10 \mathrm{dex}$ when the surface gravity is varied.

\subsection{Photometric versus spectroscopic metallicities}

For dwarf stars that are significantly more metal-rich than the $\operatorname{Sun}($ e.g. $[\mathrm{Me} / \mathrm{H}] \approx 0.2 \mathrm{dex})$, the metallicity used in the calculation of the model atmosphere is important, since it governs the line blanketing and thus the temperature structure of the model atmosphere. We may expect that final derived abundances are sensitive to this parameter. 
Following Edvardsson et al. (1993a) we decreased (and in a few cases increased) the metallicities to the values derived for $[\mathrm{Fe} / \mathrm{H}]$ in a preliminary abundance analysis and reiterated the abundance determination. (We have determined iron abundances from lines arising from both Fe I and FeII. For FeI usually more than 30 lines were analysed and for Fe II three to four lines. The formal error in the mean $[\mathrm{Fe} / \mathrm{H}]$ derived from $\mathrm{Fe}$ I for a certain star is usually smaller than 0.02 dex.) In the mean we find that we had to reduce the abundances by 0.07 dex from the initial photometric values, with a spread of $0.01 \mathrm{dex}$, Table 1. Apparently, our photometric metallicities tend to overestimate the metal content in these metal-rich stars as compared to the iron abundance derived from spectral abundance analysis, Fig. 2.

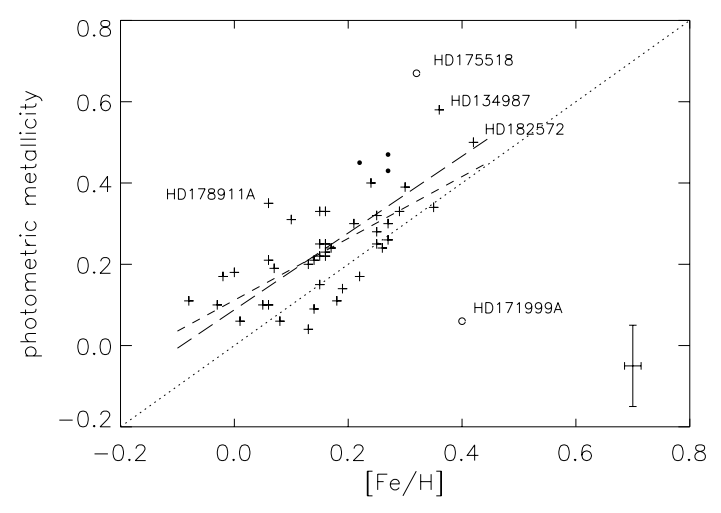

Fig. 2. Metallicities determined from photometry vs. iron abundances derived in our spectral analysis. The one-to-one relation is indicated by a dotted line and a least-square fit to the data points, taking the error in $[\mathrm{Fe} / \mathrm{H}]$ to be \pm 0.02 and the error in the photometric metallicities to be \pm 0.1 , is also shown, dashed line. The $\chi^{2}$-proabability for this fit is 0.92 . A fit made without taking the errors into account is shown by a long-dashed line. The stars from Barbuy \& Grenon (1990), •, and HD 171999A and HD 175518, denoted by $\circ$ symbols, were excluded from the fit (see Sect. 5.5)

Usually, the differences between the metallicities estimated from photometry, are close enough to those derived from spectroscopy that we have not found it necessary to reiterate the determination of effective temperature and $\log g$. (The determination of metallicity was, however, changed according to the spectroscopic result so that the final model used in the analysis had $[\mathrm{Me} / \mathrm{H}]$ consistent with the resulting spectroscopic $[\mathrm{Fe} / \mathrm{H}]$.) However, for some of the stars the photometry indicates rather extreme metallicities compared with the spectroscopic iron abundances. For HD 171999A we have only measured 6 Fe I equivalent widths (since this star was only observed with one CCD setting, see Sect. 3.1) and thus the spectroscopically determined iron abundance is not as good as for the other stars. However, we note that the line-to-line scatter is small, 0.03 dex. For HD 175518 it is questionable if its photometric metallicity is realistic. In any large catalogue there will always be a few stars with $2-3 \sigma$ errors in the photometry. Since spectroscopic iron abundances indicate a much lower metallicity this is most probably an example of that.

We have studied, for HD 175518, the effects on derived abundances if $[\mathrm{Me} / \mathrm{H}]$ is lowered to 0.2 dex as indicated by the spectroscopy, thus affecting the estimates of the rest of the fundamental parameters. The lower metallicity implies a lower effective temperature; $[\mathrm{Me} / \mathrm{H}]=0.22$ dex corresponds to a decrease of $T_{\text {eff }}$ by $\sim 200 \mathrm{~K}$. From Table 3 we see that most elements will change by $\sim 0.1$ dex and thus the star will mainly move horizontally, by $\sim 0.1$ dex, in our relative abundance diagrams. HD 175518 is an extreme case in our sample and the abundances of the other stars and general abundance trends for the whole sample should not be affected by comparable amounts.

\subsection{Effects of hyperfine structure}

Some of the elements analysed are well known to be subject to hyperfine structure. We have not taken this into account when deriving the chemical abundances. Instead, for those elements in particular we have aimed at selecting weak enough lines, so that the neglect of hyperfine structure in the abundance analysis should not affect the calculated equivalent widths and thus not the derived abundances.

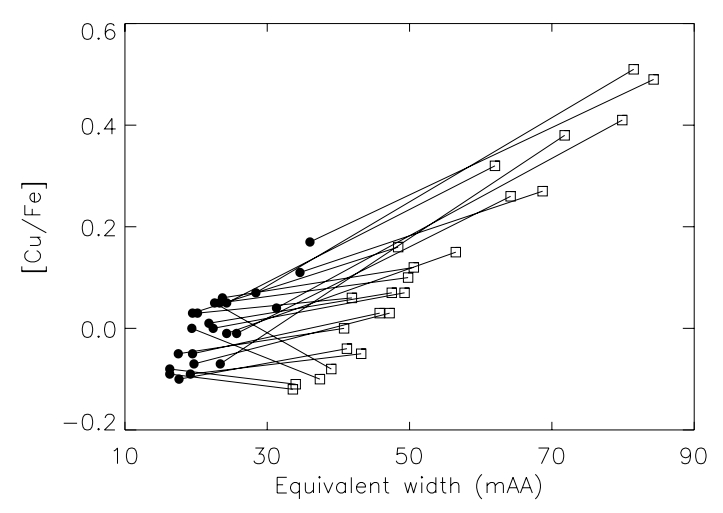

Fig. 3. Copper abundances derived from the lines at $5220 \AA$, - symbols, and $7933 \AA, \square$ symbols. The widths of these lines are 16 and $36 \mathrm{~m} \AA$, respectively, in the solar flux spectrum

Copper. Figure 3 shows copper abundances derived from the two lines used in our study as functions of equivalent width. Our data clearly show that the stronger copper line is subject to hyperfine structure and that these 
lines should be analysed using synthetic spectroscopy taking the hyperfine structure splitting into account. Results by Summers (1994) may suggest departures from LTE in the population of levels in the copper atom. We do not, however, have enough data to make further empirical investigations of such departures from LTE. We omit copper abundances from the following discussion.

Manganese and Cobalt. The manganese and cobalt lines used in this study are not saturated. When plotting abundances derived from each line as a function of equivalent width no distinct pattern was found; indicating that the omission of hyperfine structure in the treatment of the lines is not problematic. In many stars lines with equivalent widths of about 20 and $60 \mathrm{~m} \AA$, respectively, gave manganese abundances that are in excellent mutual agreement.

$r$ - and s-process elements. The lines used to derive abundances for the heavy $s$ - and $r$-process elements are sufficiently weak in our programme stars to be safely used as abundance criteria in spite of being subject to hyperfine structure. Among the light $s$-process elements we note, however, that only Y II, and maybe Y I, have lines strong enough and secure enough that derived abundances can be used with confidence. The $\log g f$-values for zirconium are very uncertain because of the faintness of the lines in the Sun.

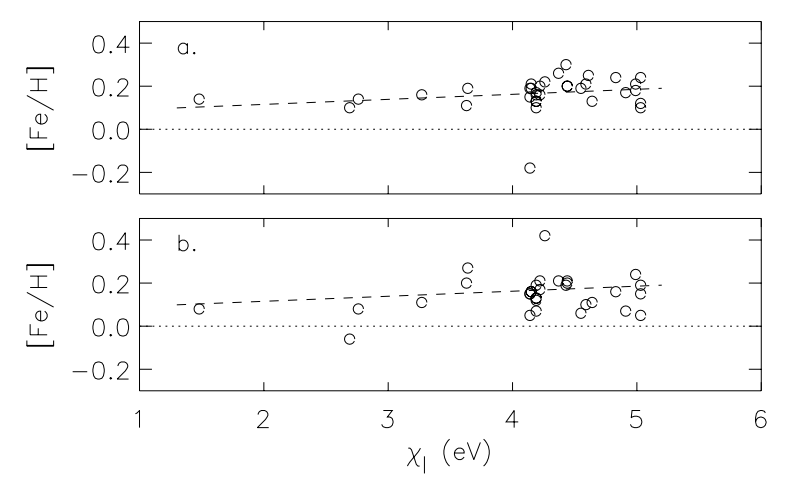

Fig. 4. Two examples of how the slope coefficients in Fig. 5 were obtained. Examples are for a) HD 91204 and b) HD 125968. The dashed lines represent linear least-square linear fits

\subsection{Checks on Boltzmann and Saha equilibria}

Deviations from the Boltzmann excitation equilibrium, which might suggest an error in the effective temperature, can be traced by studying abundances derived from individual lines as a function of the lower excitation potential for the lines. $32 \mathrm{Fe}$ I lines measured in most of the
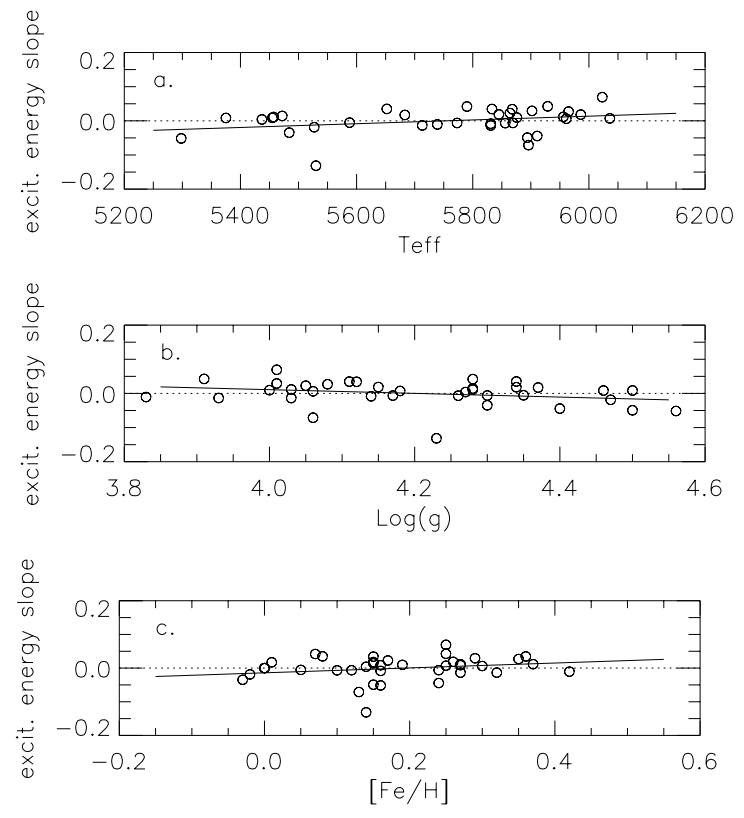

Fig. 5. The slope coefficients from the excitation energy balance diagram for each star plotted vs. effective temperature, surface gravity and spectroscopically derived iron abundances. The star with the lowest $k$ is HD 180890. Linear least square fits yield: a) $k=-0.31+5.510^{-5} \cdot T_{\text {eff }}$, b) $k=0.23-0.054 \cdot \log g$, c) $k=-0.014+0.073 \cdot[\mathrm{Fe} / \mathrm{H}]$

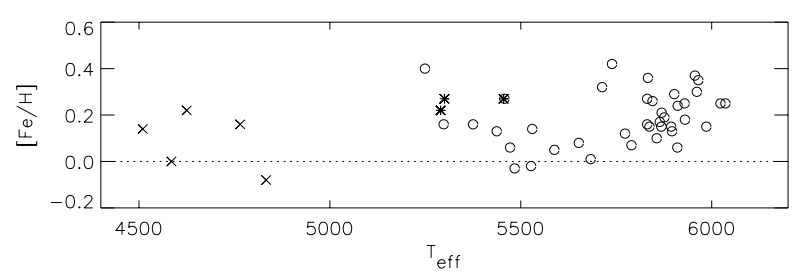

Fig. 6. Iron abundances, derived in this study, as a function of effective temperatures

stars, marked in Table 2, were selected for this purpose. For all stars (excluding four stars with too few lines observed) the abundance of each iron line was plotted as a function of the excitation energy of the lower level, $\chi_{1}$, and a least square linear fit was made to the points of the form $[\mathrm{Fe} / \mathrm{H}]=a+k \cdot \chi_{\mathrm{l}}$, Fig. 4. The mean value of the slope coefficients, $k$, is 0.00 (excluding the $\mathrm{K}$ dwarf stars), suggesting that the systematic errors in effective temperatures are small.

Next, these linear coefficients were plotted as functions of effective temperature, surface gravity and spectroscopically derived iron abundance, Fig. 5. We have compared our results with those obtained by Edvardsson et al. (1993a) (their Figs. 9a-f) and find that the two studies span the same range of excitation energy slopes, $k$. There 
are small but systematic deviations from the excitation equilibrium, varying with effective temperature. A change in $T_{\text {eff }}$ of $+100 \mathrm{~K}$ results in a $\Delta k$ of $+0.006 \mathrm{dex} \mathrm{eV}^{-1}$. The change of excitation energy slope with surface gravity estimates seems to reflect the variation of surface gravity with effective temperature (surface gravity increases as effective temperature decreases).

In many studies surface gravities are determined by requiring ionization equilibrium. This is typically made by changing the surface gravity until the iron abundances derived from Fe I and Fe II lines yield the same abundance. We have not, as discussed earlier, used this method. As we will see this has led us to discover what appears to be a case of significant overionization in $\mathrm{K}$ dwarf stars and an opposite smaller effect for the hotter stars (see Fig. 7).

What would the effects be if we assumed ionization equilibrium, and adjusted the surface gravities accordingly? We can estimate changes in the stellar abundances from the results of Table 3 and Table 11. From this we find that half of the stars should have their surface gravities increased by $0.25-0.35$ dex to achieve ionization equilibrium for iron. This means that the the iron abundance will change with $\approx-0.03$. Abundances of other elements will change with similar amounts but with differing signs, which means that for some elements $[\mathrm{X} / \mathrm{Fe}]$ will change by up to 0.1 dex and for others not at all. However, we note that the oxygen abundances are very sensitive to the surface gravity and may change by up to 0.2 dex. As a comparison we estimate the maximum error in the derived oxygen abundance caused by incorrectly set continua to be less than 0.1 dex.

An adjustment downwards of the gravities by about 0.3 dex would increase the conflict with the gravity estimates from the Ca I $6162 \AA$ line wings. We consider such a revision less probable.

\subsection{K dwarf stars - overionization}

Our results admit a comparison for five elements (scandium, vanadium, chromium, iron and yttrium) of abundances derived from ions to abundances derived from the corresponding atom, as function of effective temperature, within a rather wide range of effective temperature.

We find an apparent overionization as compared to expectations from LTE calculations for the five $\mathrm{K}$ dwarf stars in our study, Fig. 7. Our results are at first sight unexpected, especially for the two $\mathrm{K}$ dwarf stars with iron abundances of $\sim 0.3$ dex relative to the solar iron abundance, as derived from Fe I lines. In stars more metalrich than the Sun the photoionizing non-local UV-flux is more efficiently blocked than in more metal-poor stars. However, as discussed in Sect. 5.9, the stronger blocking may be more than compensated by the increased temperature gradient which may enhance the non-locality of the radiation field.
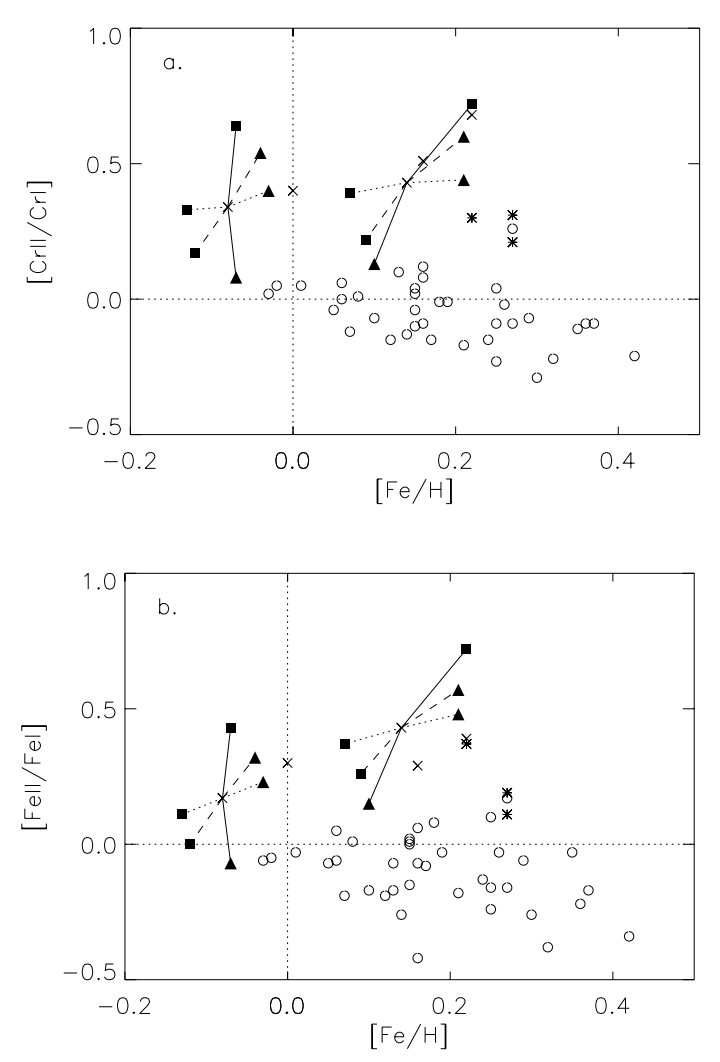

Fig. 7. Abundance ratios of chromium and iron as functions of $[\mathrm{Fe} / \mathrm{H}]$. $[\mathrm{Cr} \mathrm{II} / \mathrm{Cr} \mathrm{I}]$, denotes $[\mathrm{Cr} / \mathrm{H}]_{\mathrm{LTE}}$ as determined from Cr II lines minus $[\mathrm{Cr} / \mathrm{H}]_{\text {LTE }}$ as determined from $\mathrm{Cr}$ I lines, and similarly for iron. Results for chromium are shown in panel a) and for iron in panel $\mathbf{b}) \times$ symbols denote the $\mathrm{K}$ dwarf stars and $*$ symbols denote the stars from Barbuy \& Grenon (1990). We exemplify, with HD 61606A $([\mathrm{Fe} / \mathrm{H}]=-0.08)$ and HD $103932([\mathrm{Fe} / \mathrm{H}]=0.16)$, how the resulting loci of the $\mathrm{K}$ dwarf stars will be shifted in the diagram if the parameters of the stellar model atmospheres are changed; changes of $T_{\text {eff }} \pm$ $200 \mathrm{~K}$ (solid lines), $\log g \pm 0.4 \mathrm{dex}$ (dashed lines) and $[\mathrm{Fe} / \mathrm{H}] \pm$ 0.2 dex (dotted lines) are shown. Filled triangles denote increased values of the respective parameters while filled squares denote decreased values

We have carefully inspected the Cr II and Fe II lines in the $\mathrm{K}$ dwarf spectra and excluded all lines which may be subject to severe blends in these cool stars. For Fe II we used the lines at 6456.39 and $6516.39 \AA$ and for Cr II the lines at 5305.86 and $5310.69 \AA$. In spite of using blend free lines the apparent overionization remains. The internal consistency between abundances derived from the two Fe II lines is very good and this is also the case for Cr II.

For scandium, vanadium and yttrium blends remain a possible source of error, but the similarity with the trends for iron and chromium suggests a common cause of the apparent overionization for all these elements.

The iron abundances derived from the atom show no obvious trend with effective temperature, see Fig. 6 . 
Table 4. The table shows results of different derivations of the effective temperatures for the five $\mathrm{K}$ dwarf stars; in the second column as derived from the calibration by Olsen (1984), and which are used in this study; in the third spectral classes; in the fourth and fifth differences between effective temperature derived in this work and from MK class calibrations by Bell \& Gustafsson (1989) and Johnson (1966); in the sixth and seventh $B-V$ and effective temperatures derived from $B-V$ using the calibration of Johnson (1966). The three last columns contain effective temperatures derived by other authors, Neff et al. (1995), Morell (1994) and Arribas \& Martinez Roger (1989)

\begin{tabular}{|c|c|c|c|c|c|c|c|c|c|}
\hline \multirow[b]{2}{*}{ ID } & \multirow{2}{*}{$\frac{u v b y-\beta}{T_{\mathrm{eff}}}$} & \multirow{2}{*}{$\begin{array}{r}\text { Spectral } \\
\text { class }\end{array}$} & \multirow{2}{*}{$\begin{array}{r}\begin{array}{r}\text { Bell \& } \\
\text { Gustafsson }\end{array} \\
\Delta T_{\text {eff }}\end{array}$} & \multirow{2}{*}{$\begin{array}{r}\text { Johnson } \\
\Delta T_{\text {eff }}\end{array}$} & \multicolumn{2}{|c|}{ Johnson } & \multirow{2}{*}{$\begin{array}{r}\begin{array}{c}\text { Neff } \\
\text { et al. }\end{array} \\
T_{\text {eff }}\end{array}$} & \multirow{2}{*}{$\begin{array}{r}\text { Morell } \\
T_{\text {eff }}\end{array}$} & \multirow{2}{*}{$\begin{array}{c}\begin{array}{c}\text { Arribas \& } \\
\text { Martinez Roger }\end{array} \\
T_{\text {eff }}\end{array}$} \\
\hline & & & & & $B-V$ & $T_{\text {eff }}$ & & & \\
\hline HD 32147 & 4625 & $\mathrm{~K} 3 \mathrm{~V}$ & +200 & +100 & 1.06 & 4619 & 4570 & & $4670 \pm 150$ \\
\hline HD $61606 \mathrm{~A}$ & 4833 & $\mathrm{~K} 2 \mathrm{~V}$ & +300 & +100 & 0.96 & 4863 & & & \\
\hline HD 103932 & 4510 & $\mathrm{~K} 5 \mathrm{~V}$ & -300 & -100 & 1.12 & 4473 & & & \\
\hline HD $131977 \mathrm{~A}$ & 4585 & $\mathrm{~K} 4 \mathrm{~V}$ & -100 & 0 & 1.10 & 4522 & 4575 & 4570 & \\
\hline HD 136834 & 4765 & $\mathrm{~K} 3 \mathrm{~V}$ & +100 & 0 & 1.00 & 4765 & & & \\
\hline
\end{tabular}

A probable reason for these effects is overionization (see Sect. 5.9). Before discussing this, however, we shall explore the possibility that errors in the temperature scale could also contribute significantly.

\subsubsection{Errors in effective temperatures for $\mathrm{K}$ dwarf stars}

The calibration of the photometry in Olsen (1984) is, for $b-y>0$. 510 , based on a sample of $15 \mathrm{~K}$ and $\mathrm{M}$ dwarf stars using stellar parameters from Cayrel de Strobel \& Bentolila (1983). The K and M dwarf stars in Olsen's sample span a small range in $\delta m_{1}$ and $\delta c_{1}$. This is reflected in the change in the calibration at $b-y=0.510$. For $b-y>0.510$ the calibration is degenerate in metallicity. Olsen (1984) quotes an error of $\sim 100 \mathrm{~K}$ for the effective temperature as derived from $b-y$.

Additional photometry is scarce for our $\mathrm{K}$ dwarf stars. However, $U B V$ photometry exists and we have checked our effective temperatures using the calibration of $B-V$ by Johnson (1966), Table 4. These effective temperatures agree well with those obtained from $u v b y-\beta$ photometry. We note, however, that the increased blocking for metalrich stars, as compared to the calibration stars used by Johnson (1966), may cause the present effective temperatures to be underestimated. We have also derived (crude) effective temperatures from calibrations of the MK classification. Comparing the effective temperatures derived from photometry with calibrations of effective temperatures as functions of spectral classification (Bell \& Gustafsson 1989 and Johnson 1966) we estimate $T_{\text {eff-values that deviate as }}$ compared with our standard values as indicated in Table 4, Cols. 4 and 5.

We have searched the literature for independent derivations of the effective temperature for these stars. Those found agree well with the photometric results, Table 4. As described earlier we have also used the excitation energy balance to check our effective temperatures. A change in effective temperature of $+200 \mathrm{~K}$ for HD 32147 changed the slope of the least-square fit to the data points in the diagram abundance-versus-excitation energy from +0.04 to +0.00 . A change of $-200 \mathrm{~K}$ brought about a similar change but in the opposite direction. Thus, the excitation equilibrium indicates that $+200 \mathrm{~K}$ is an acceptable change of the effective temperature for this star. The line-to-line scatter in derived abundances from Fe I lines for this star is among the largest, \pm 0.13 . The same changes in effective temperatures give similar values for HD 61606 A. Since the other three $\mathrm{K}$ dwarf stars are in the same effective temperature range and have similar surface gravities as the two stars discussed here, and since HD 32147 is the most metal-rich and HD 61606A is the most metal-poor of the five $\mathrm{K}$ dwarf stars, changes in the fundamental parameters of the remaining three stars will produce similar changes in abundances.

To conclude, a change in effective temperature of +200 to $+400 \mathrm{~K}$ may be allowed as judged from the excitation equilibrium. As is obvious from Fig. 7, a change of this order of magnitude would restore the LTE ionization balance for iron and chromium. The large line-to-line scatter in derived abundances for lines with high excitation energies makes attempts to derive effective temperatures from excitation equilibria very dependent on one or two points in the lower end of the excitation energy range, spanned by the lines as illustrated in Fig. 4. Effective temperatures derived in other studies and from $B-V$ colours deviate by less than this from our values.

As already noted the surface gravities for the $\mathrm{K}$ dwarf stars seem rather well determined, see Table 1 . We also note that our $\log g$ value for HD 131977A agrees well with that given by Morell (1994). We conclude that realistic errors in $\log g$ are not enough to account for the departure from ionization equilibrium.

To conclude, we cannot from our analysis exclude that the apparent pattern of overionization, at least partially, is due to a temperature scale that is several hundred $\mathrm{K}$ too low. However, our analysis, together with evidence from 
other studies, suggest that deviations from LTE is a more plausible cause for the effects.

\subsection{Non-LTE}

No detailed study has been devoted to the non-LTE effects on abundance determinations for metal-rich dwarf stars, cooler than the Sun. A general result of the available studies for solar-type stars is, however, that several different effects are at play and may counteract each other, and this makes all extrapolation to the present study of metal-rich dwarf stars from studies of other types of stars or studies of other elements questionable.

Among the significant effects are (cf. Bruls et al. 1992) resonance line scattering, photon suction, ultraviolet overionization, (infra)red over-recombination and optical line pumping. The resonance-line scattering effects, in which photon losses cause the source functions of resonance lines to drop far below the Planck function at depths greater than those where the line optical depth is unity, may lead to severe overestimates of abundances - e.g., Carlsson et al. (1994) find that in Li-rich cool stars the Li abundance may well be overestimated by a factor of 3 as a result of this.

Photon suction may, for metal-rich cases in particular, lead to overpopulation of, e.g., the ground state and thus inhibit the effects of overionization. This is the result of a compensation of photon losses in the upper photosphere in resonance lines, as well as in connected ladders of transitions, by a downward population replenishment flow from the continuum reservoir. It is of great significance for atoms with a majority of corresponding ions and with pronounced cascade ladders. For complex atoms it should be of greatest significance for the high-lying levels that thus can compensate population depletion processes at lower excitation energy, e.g. caused by overionization.

The ultraviolet overionization has been a major worry in analyses of late type stellar spectra for two decades. It arises because the mean intensity $J_{\nu}$ drops below the Planck function $B_{\nu}$ in the line-forming regions of the atmosphere on the blue side of the spectrum peak. Overionization is known to occur for Fe I in the Sun from levels a few electron volts below the continuum (see Rutten 1988 and references therein) and may more or less effect other metals as well (see, e.g., Baumueller \& Gehren 1996; Bruls 1993). Overionization was suggested by Auman \& Woodrow (1975) to be significant for a number of elements with lower ionization energies in cool stars. Major problems in modeling it are, however, the difficulties in predicting the ultraviolet flux of late-type stars with the crowding of spectral lines and the possible existence of an "unknown opacity" (cf., e.g. Gustafsson 1995), as well as the dependence of the results of the notoriously uncertain collision cross sections, e.g. for collisions with $\mathrm{H}$ atoms (cf. Steenbock \& Holweger 1984). Empirically, Mäckle et al. (1975) and Ruland et al. (1980), found a ten- dency for the low-excitation lines $(\chi \sim 2 \mathrm{eV})$ of Fe I and $\mathrm{Ti} \mathrm{I}$ in $\mathrm{K}$ giant star spectra to give systematically lower abundances than the high-excitation lines $(\chi \sim 4.5 \mathrm{eV})$. The abundance difference was typically found to be 0.15 dex. Subsequently, Steenbock (1985) succeeded in reproducing this result with statistical-equilibrium calculations. He found the effect to mainly reflect an overionization in upper layers (notably $\tau_{5000}<-2$ ) of the atmospheres, where the low excitation lines are formed. The effect is much smaller in the solar spectrum, leading to systematic errors in a differential analysis where red giants are compared with the Sun.

For metal-rich stars, the blocking by the crowd of spectral lines in the ultraviolet could be expected to - at first sight - strongly reduce the overionization effect, but this may be compensated for by a steeper temperature gradient in their atmospheres as a result of line blanketing effects, as in the case of Li I, Carlsson et al. (1994), or of Ca I, Drake (1991). The latter study is particularly instructive for judging the results of the present investigation. Drake finds that for $\mathrm{G}$ and $\mathrm{K}$-type stars the overionization effects on Ca I abundances increase with decreasing effective temperature, with increasing acceleration of gravity and with increasing metallicity. At least the last two results may seem contrary to intuition. They reflect the significance of $\mathrm{H}$ I absorption shortwards of the Balmer discontinuity, which blocks more of the ionizing UV flux for the giants than for the dwarfs, and the afore-mentioned effects of metal-line blanketing on the temperature structure. For the $\mathrm{K}$ dwarf stars the effects on Ca I abundances may, according to Drake's results, well result in an underestimate by a factor of two or more if LTE is assumed.

Over-recombination is important for photoionization transitions from levels close to the continuum (i.e. in the infrared), since for them the angle-averaged $J_{\nu}$ may drop below the local Planck function deep in the photosphere. This may produce net recombinations, and overpopulation of the upper levels.

Optical (ultraviolet) pumping occurs in strong lines, e.g. the resonance lines and is analogous to overionization in that it is driven by $J_{\nu}>B_{\nu}$. It is important, not the least when it occurs in ultraviolet resonance lines and excites the atoms to states which may be much more easily photoionization due to a much richer radiation fields available at longer wavelengths, as was early suggested by Aumann \& Woodrow (1975). This is most important for trace elements, and for metal-poor stars.

The complex interplay between these different mechanisms affects most levels of the atom, at great atmospheric depths, for atoms where the strong lines get efficiently optically thin in the photosphere, i.e. for relatively rare elements like the alkalis, while for the more abundant atoms like $\mathrm{Fe}$ and $\mathrm{Mg}$ the stronger, e.g. resonance, transitions are in detailed balance through most of the photosphere. For these, the relatively simple overionization phenomenon is probably dominating, except for transitions very close to 
the continuum for which e.g. photon suction may be significant.

In a recent study Gratton et al. (in prep.) have used detailed statistical-equilibrium calculations to explore the departures from LTE for solar-type dwarfs as well as for red giants of different metallicities, and their effects on abundance determinations for $\mathrm{O}, \mathrm{Na}, \mathrm{Mg}$, and $\mathrm{Fe}$. These authors find relatively small effects for stars cooler than the Sun for O I - LTE abundances from the IR triplet lines should be corrected downwards by less than 0.1 dex for stars with $T_{\text {eff }} \lesssim 6000 \mathrm{~K}$. For Na I the subordinate lines are weakened by overionization and cascade by about 0.1 dex for the solar-type dwarfs. The dominating effect for $\mathrm{Mg} \mathrm{I}$ is overionization, and the non-LTE abundance corrections are thus generally positive. Typically the corrections are 0.1 dex in the dwarf stars. For Fe I, where again overionization is dominating the abundance corrections $\simeq 0.1$ dex. Most of these effects are found to be greater for $T_{\text {eff }}>6000 \mathrm{~K}$. It should be noted, however, that models for metal-rich dwarfs with $T_{\text {eff }}<5000 \mathrm{~K}$ were not included in this study.

Summing up the discussion of non-LTE we conclude that the effects on abundances are expected to be mainly due to overionization for most of the elements. For the alkali atoms, as well as for the rare earths, more complex effects may also be significant. Typically, errors of about 0.1 dex may be expected in the differential results but the complexity of the interplay between different effects, and in particular the results obtained by Drake (1991) for Ca I, suggest that greater effects may be present, in particular for the metal-rich $\mathrm{K}$ dwarf stars.

\subsection{Collecting errors}

We have shown that errors in fundamental parameters give errors in mean resulting abundances of less than 0.1 dex. For elemental abundances derived from several lines this may be the dominating error, while for abundances derived from one single line errors due to blends and fitting of continua may be the main contributors to the overall error. Deviations from LTE in the excitation and ionization balance may also be of importance, probably more so for abundances based on few lines, in particular for the $\mathrm{K}$ dwarf stars. We collect our best estimates of errors due to different sources in Table 5 .

Table 5. The effects of error sources explored in this work on estimates of abundances relative to the Sun

\begin{tabular}{lr}
\hline Source of error & Error in resulting relative abundance \\
\hline Measurement of $W_{\lambda}$ & negligible \\
Continuum fitting & $<0.09 \mathrm{dex}$, usually $0.05 \mathrm{dex}$ \\
$T_{\text {eff }}, \log g$ & $<0.1 \mathrm{dex}$ \\
Non-LTE effects & $0.1-0.2 \mathrm{dex} ?$ \\
Oscillator strengths & $<0.1 \mathrm{dex}$ \\
\hline
\end{tabular}

\subsection{Comparison of results for stars in common with other studies}

The majority of our stars have not been studied before through spectroscopic abundance analysis.

Table 6. Comparison of iron abundances between our work and the abundances quoted in the catalogue by Cayrel de Strobel et al. (1997). The second column gives our results and the third the mean, and the spread, of the iron abundances given by Cayrel de Strobel et al. In the fifth give the number of derivations used. A straight mean has been taken to represent the mean abundance from the catalogue

\begin{tabular}{lcccc}
\hline ID & {$[\mathrm{Fe} / \mathrm{H}]$} & $\langle[\mathrm{Fe} / \mathrm{H}]\rangle$ & $\pm \mathrm{s}$ & $\#$ \\
\hline HD 30562 & $0.19 \pm 0.09$ & 0.14 & 0.0 & 2 \\
HD 32147 & $0.22 \pm 0.13$ & 0.02 & 0.0 & 2 \\
HD 67228 & $0.16 \pm 0.08$ & 0.05 & & 1 \\
HD 131977A & $0.00 \pm 0.12$ & 0.01 & & 1 \\
HD 144585 & $0.27 \pm 0.05$ & 0.23 & & 1 \\
HD 182572 & $0.42 \pm 0.05$ & 0.32 & 0.14 & 7 \\
HD 186427 & $0.12 \pm 0.05$ & 0.06 & 0.04 & 5 \\
\hline
\end{tabular}

For those of our stars (HD 30562, HD 32147, HD 67228, HD 1319777, HD 182572, HD 186427) that are in the catalogue by Cayrel de Strobel et al. (1997) the agreement between iron abundances derived in this study and those listed in the catalogue is good, cf. Table 6 .

HD 32147 has been given much attention in the discussion of Super Metal Rich (SMR) stars. SMR stars have been defined as stars with $[\mathrm{Fe} / \mathrm{H}]<0.2$ dex (for a discussion and references on SMR stars see Taylor 1996). Low resolution work and photometric determinations of $[\mathrm{Fe} / \mathrm{H}]$ have been carried out for this stars, but this is, to our knowledge, the first high dispersion analyses of the star. Our $[\mathrm{Fe} / \mathrm{H}]$ of 0.28 dex implies that this star is really an SMR star.

Our results for HD 182572 are compared with the results of the detailed analysis by McWilliam (1990) in Table 7. We note that considerable discrepancies remain even after correcting for the difference in effective temperatures.

We have analysed three stars previously studied by Edvardsson et al. (1993a). The results are compared in Table 8. For HD 30562 and HD 144585 the results agree to within the errors quoted. The results for HD 67228 show larger discrepancies than the other stars for magnesium, silicon, calcium and iron. A higher microturbulence parameter, as used in Edvardsson et al. (1993a), would decrease our results by 0.01 or 0.02 dex (ionized iron by 0.04 dex). Edvardsson et al. (1993a) find that a change in $T_{\text {eff }}$ by $+100 \mathrm{~K}$ gives an iron abundance 0.06 higher for their stars. Thus, the increase of $52 \mathrm{~K}$ needed to transform the results of Edvardsson et al. (1993a) to our temperature scale means an increase of their iron abundance by 
Table 7. Comparison of abundances, $[\mathrm{X} / \mathrm{H}]$, derived for HD 182572 (HR 7373) in our study and by McWilliam (1990). McWilliam uses $\left(T_{\text {eff }} / \log g /[\mathrm{Fe} / \mathrm{H}] / \xi_{\mathrm{t}}\right)=(5380 / 3.92 / 0.15 / 1.9)$ and we $(5739 / 3.83 / 0.42 / 1.9)$. The results by McWilliam have been scaled to the same solar abundances as we use, Table 2, (this is most important for iron). In the last column our values are scaled to the effective temperature used by McWilliam

\begin{tabular}{lrrr}
\hline & Mc William & \multicolumn{2}{c}{ This work } \\
& & $5739 \mathrm{~K}$ & $5380 \mathrm{~K}$ \\
\hline Fe I & 0.31 & 0.42 & 0.24 \\
Si I & 0.28 & 0.51 & 0.47 \\
Ca I & -0.11 & 0.42 & 0.21 \\
Sc II & 0.14 & 0.36 & 0.36 \\
Ti I & -0.02 & 0.50 & 0.21 \\
V I & -0.02 & 0.44 & 0.12 \\
Co I & 0.18 & 0.58 & 0.29 \\
Ni I & 0.00 & 0.46 & 0.24 \\
Eu II & 0.18 & 0.13 & 0.13 \\
\hline
\end{tabular}

Table 8. The first column for each star contains the result of this work, the second the difference between the two studies, Diff $=$ this work - Edvardsson et al. (1993a). Stellar parameters used by Edvardsson et al. are for HD $30563\left(T_{\text {eff }} / \log g /[\mathrm{Fe} / \mathrm{H}]\right)=(5886 / 3.98 / 0.17)$, for HD 67228 $(5779 / 4.20 / 0.04)$ and for HD 144585 (5831/4.03/0.23). The values of the parameters used in this study may be found in Table 1

\begin{tabular}{|c|c|c|c|c|c|c|}
\hline & HD & $\begin{array}{r}30562 \\
\text { Diff. }\end{array}$ & HD & $\begin{array}{r}67228 \\
\text { Diff. }\end{array}$ & HD & $\begin{array}{r}44585 \\
\text { Diff. }\end{array}$ \\
\hline$[\mathrm{O}$ I] & & & 0.21 & & & \\
\hline $\mathrm{NaI}$ & 0.21 & +0.01 & 0.23 & +0.06 & 0.36 & \pm 0.00 \\
\hline Mg I & 0.33 & -0.01 & 0.23 & +0.08 & & \\
\hline $\mathrm{Al}$ I & 0.25 & -0.07 & 0.22 & +0.03 & & \\
\hline Si I & 0.21 & -0.02 & 0.27 & +0.11 & 0.24 & -0.03 \\
\hline Ca I & 0.17 & -0.01 & 0.15 & +0.12 & 0.24 & -0.03 \\
\hline Ti I & 0.13 & -0.02 & 0.09 & +0.02 & 0.29 & +0.02 \\
\hline $\mathrm{Fe} I$ & 0.19 & +0.05 & 0.16 & +0.12 & 0.27 & +0.04 \\
\hline Fe II & 0.16 & +0.10 & 0.22 & +0.06 & 0.11 & -0.05 \\
\hline $\mathrm{Ni}$ I & 0.17 & \pm 0.00 & 0.14 & -0.01 & 0.32 & +0.06 \\
\hline Y II & 0.09 & & 0.12 & \pm 0.00 & 0.06 & +0.06 \\
\hline Nd II & & & -0.10 & & -0.14 & -0.06 \\
\hline
\end{tabular}

0.03 dex. Also the silicon and calcium abundances are affected in the same way as iron while all the other abundances remain as before, within the errors, in the two studies. The $\log g f$-values agree well (0.06 difference, we have the higher value). The spectrum we have obtained for this star is of high quality $(S / N \sim 200)$. We do not find the discrepancy between the two studies alarming. HD 67228 has also been studied by Andersen et al. (1984) in a study on lithium isotope ratios in $\mathrm{F}$ and $\mathrm{G}$ dwarf stars. They derive an $[\mathrm{Fe} / \mathrm{H}]$ of 0.05 from spectral lines using a model with $\left(T_{\text {eff }} / \log g /[\mathrm{Fe} / \mathrm{H}]\right)=(5850 / 4.2 / 0.05)$.
Table 9. Comparison of derived abundances, $[\mathrm{X} / \mathrm{H}]$, between Friel et al. (1993) and this work for HD 186427, 16 Cyg B. Friel et al. use the following stellar parameters $\left(\mathrm{T}_{\text {eff }} / \log g /[\mathrm{Fe} / \mathrm{H}]\right)=(5770 / 4.30 / 0.05)$ and we use $(5773 / 4.17 / 0.12)$

\begin{tabular}{lllll}
\hline & Na I & Al I & \multicolumn{1}{l}{ Si I } & Ca I \\
\hline Friel et al. & 0.07 & 0.12 & $0.06 \pm 0.03$ & $0.07 \pm 0.05$ \\
This work & $0.13 \pm 0.04$ & $0.12 \pm 0.04$ & $0.10 \pm 0.04$ & $0.02 \pm 0.09$ \\
\hline & Ti I & Fe I & Fe II & Ni I \\
\hline & $0.10 \pm 0.04$ & $0.05 \pm 0.03$ & $0.02 \pm 0.04$ & $0.05 \pm 0.03$ \\
& $0.07 \pm 0.10$ & $0.05 \pm 0.05$ & $-0.06 \pm 0.04$ & $0.04 \pm 0.10$ \\
\hline
\end{tabular}

Two stars from our sample, HD 30562 and HD 67228, were recently analysed in detail by Tomkin et al. (1997) on the basis of different spectra; however, obtained with the same instrument and analysed independently with model atmospheres computed with the same computer program. The results are compared with those of our analyses in Table 10. In view of the errors in these analyses we find the agreement satisfactory.

HD 186427 (16 Cyg B) have been extensively studied, in particular in connection with searches for solar twins. A recent spectroscopic study has been performed by Friel et al. (1993). The results are in good agreement, Table 9. However, we find lower iron abundances derived from Fe II lines than Friel et al. (1993) do. This difference is probably mainly due to the different surface gravities used.

To conclude, we find that, for those few stars in our programme in common with other studies, abundances are rather well reproduced. This gives confidence when we now apply our results to the exploration of the chemical evolution of the Galaxy.

\section{Abundance results}

Our abundance results are presented in Table 11 and relative abundances are plotted in Figs. 8, 9, 13 and 6. Below, we shall compare our results with model calculations for the galactic chemical evolution. Sometimes, we shall also quote results of other studies, in particular that of Edvardsson et al. (1993a) (which is grossly compatible in terms of calibration and methods with the present one), but also with others, in particular for Population II dwarf stars.

Comparison of stellar abundance results from different studies may not always be straightforward. There are many inconsistencies that may confuse the interpretation of such comparisons. The usage of different lines for the abundance analysis, different stellar parameters, different model atmospheres, and, if the study is differential to the 
Table 10. Comparison between results of our analyses for HD 30592 and HD 67228, with the results of Tomkin et al. (1997). The second and fourth column give the results of this work, while the third and fifth give the difference between this work and that of Tomkin et al. Stellar fundamental parameters of Tomkin et al. were $\left(T_{\text {eff }} / \log g /[\mathrm{Me} / \mathrm{H}] / \xi_{\mathrm{t}}\right)=$ $(5930 / 4.20 / 0.21 / 1.55)$ and $(5835 / 4.10 / 0.09 / 1.75)$ for HD 30592 and HD 67228, respectively. Our parameters are given in Table 1

\begin{tabular}{|c|c|c|c|c|}
\hline & HD & $\begin{array}{r}30562 \\
\text { Diff. }\end{array}$ & HD & $\begin{array}{r}67228 \\
\text { Diff. }\end{array}$ \\
\hline [O I] & & & 0.21 & 0.01 \\
\hline $\mathrm{Na} \mathrm{I}$ & 0.21 & -0.02 & 0.23 & +0.03 \\
\hline Mg I & 0.33 & 0.12 & 0.23 & 0.09 \\
\hline $\mathrm{Al} \mathrm{I}$ & 0.25 & -0.01 & 0.22 & 0.03 \\
\hline Si I & 0.21 & -0.04 & 0.27 & 0.12 \\
\hline Ca I & 0.17 & -0.06 & 0.15 & 0.08 \\
\hline Sc II & 0.31 & 0.06 & 0.33 & 0.16 \\
\hline Ti I & 0.13 & -0.12 & 0.09 & -0.02 \\
\hline V II & 0.10 & -0.16 & 0.12 & 0.01 \\
\hline Cr I & 0.18 & -0.02 & & \\
\hline Cr II & 0.17 & -0.05 & 0.18 & 0.02 \\
\hline $\mathrm{Fe} I$ & 0.19 & -0.02 & 0.16 & 0.07 \\
\hline Fe II & 0.16 & -0.04 & 0.22 & 0.10 \\
\hline Ni I & 0.17 & -0.08 & 0.14 & 0.01 \\
\hline YI I & 0.09 & -0.12 & 0.12 & 0.06 \\
\hline Zr I & 0.65 & 0.39 & & \\
\hline Eu II & 0.14 & -0.17 & & \\
\hline
\end{tabular}

Sun, different solar model atmospheres; all of these may lead to offsets and may cause the compiled data to show trends which are unreal. Nevertheless, below we shall compare our abundances with results from other studies to put our results into a broader picture of the galactic chemical evolution.

\subsection{Iron}

In observational studies of galactic chemical evolution iron is often used as the reference element. The reason for this is twofold; iron is believed, but this is debated, to be a fair chronometer for the nucleosynthesis in the Galaxy, and the spectra of dwarf stars show many iron lines, easy to measure. The evolutionary picture for iron is complicated by the fact that iron is produced in both core collapse and type Ia supernovae. From this point of view oxygen, which is only produced in core-collapse supernovae, may be preferable as reference element. However, as we will discuss in Sect. 6.2, oxygen abundances are not trivial to derive. We will therefore conform with common practise and use iron as reference element.

Our resulting iron abundances are well determined with a line-to-line scatter in $[\mathrm{Fe} / \mathrm{H}]$ of typically $0.09 \mathrm{dex}$ and a formal error in the mean iron abundance of typically less than 0.02 dex for each star. We find that $[\mathrm{Fe} / \mathrm{H}]$ does not vary with $V_{\mathrm{LSR}}$, i.e. $R_{\mathrm{m}}$, Fig. 9 . This is an important observation when we consider other elemental abundances relative to Fe later, cf. Sect. 6.3, and a first indication that the mixing of gas over the $\Delta R_{\mathrm{m}}$ spanned has been quite efficient. The five $\mathrm{K}$ dwarf stars show a similar behaviour as the rest of the sample.

\subsection{Oxygen}

Oxygen is the third most abundant element in stars and therefore plays a significant role for stellar opacities and energy generation. Therefore, determination of stellar ages depends strongly on the assumed initial oxygen abundance in the star, see e.g. VandenBerg (1992). Oxygen abundances also affect the determination of time-scales in the galactic chemical evolution and star-formation rates. Thus, it is important to know the amount of oxygen throughout the history of the Galaxy.

We have studied three oxygen criteria; the forbidden line at $6300 \AA$, the $6158 \AA$ line and the $7774 \AA$ triplet. These criteria are commonly used; however, discrepancies between the abundances derived from the different criteria for the Sun, as well as for other late-type stars, have prevailed in spite of much work (cf. e.g. Eriksson \& Toft (1979) and Kiselman (1993) and references therein). NonLTE and granulation are two proposed sources of the discrepancy between abundances derived from the [O I] line and from the triplet lines.

The formation of the [O I] line is expected not to be subject to departures from LTE. The lower level of the transition is the ground level of the atom and the majority of the oxygen should be found in the ground state of the atom under solar photospheric conditions. There are, however, suspicions that the analysis of the line might be subject to systematic errors due to the adoption of plane parallel stellar atmosphere models since granulation effects are not taken into account in these models, Kiselman \& Nordlund (1995).

Edvardsson et al. (1993a) found a correlation between oxygen abundances derived from the $[\mathrm{OI}]$ line and the abundances derived from the triplet lines as well as a correlation with the abundance derived from the $6158 \AA$ line. These relations are shown, together with our data, in Fig. 11. We do not find a clear correlation between abundances derived from $[\mathrm{O} \mathrm{I}]$ and the abundances derived from the $7774 \AA$ triplet and $6158 \AA$ lines for our stars. This circumstance, and the fact that the [O I] line is not expected to be affected by departures from LTE, lead us to use only the abundance derived from the [O I] line in our analysis.

When comparing our results with those of Nissen \& Edvardsson (1992) we find in general a higher oxygen abundance. This discrepancy can be understood: Nissen \& Edvardsson (1992) have used an oscillator strength of -9.75 (Lambert 1978) while ours is -9.84 . This means that our abundances should be scaled down by 0.09 dex 
Table 11. Elemental abundances our programme stars. For each star and each ion the derived elemental abundance, the line-to-line scatter (the error in the mean $=$ line-to-line scatter $/ \sqrt{n}_{\text {lines }}$ ) and the number of lines used in the analysis are given. The table is continued on the following pages. This table is only published electronically
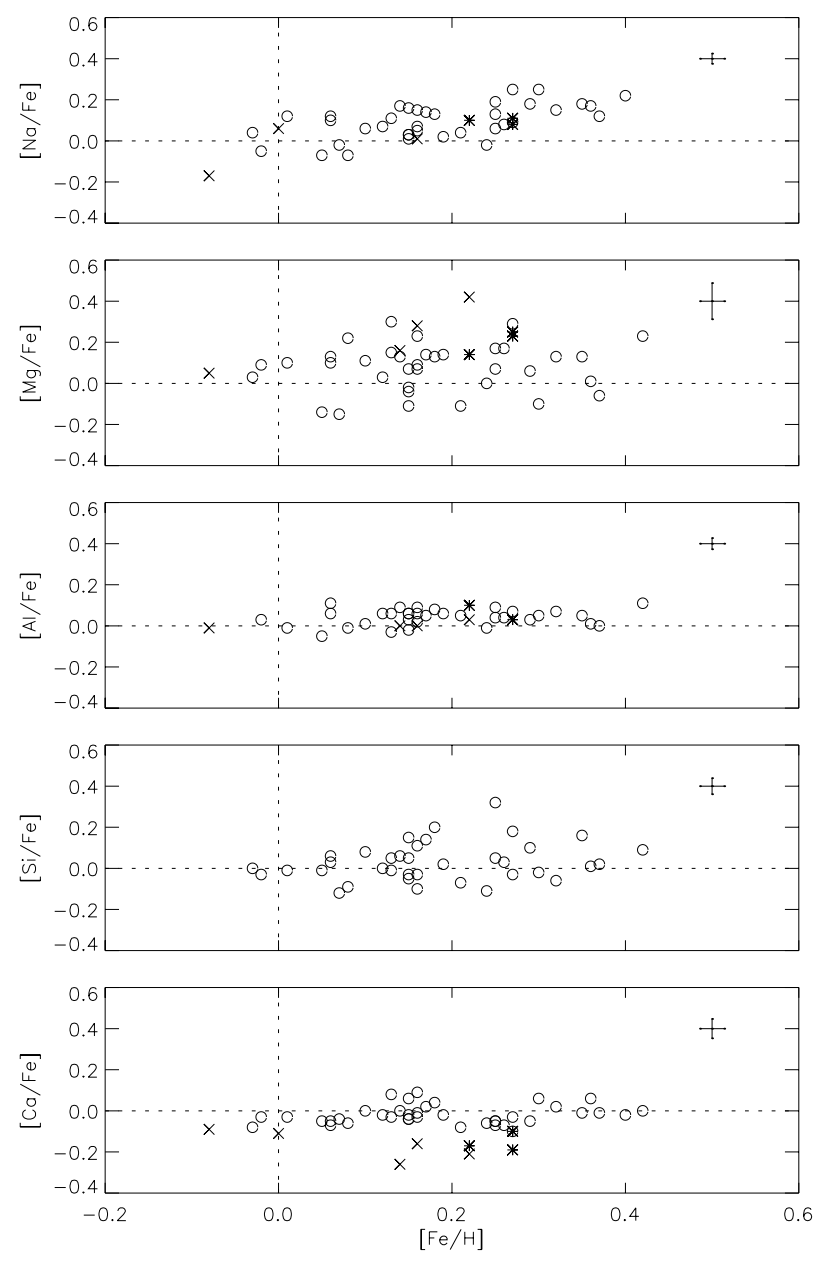
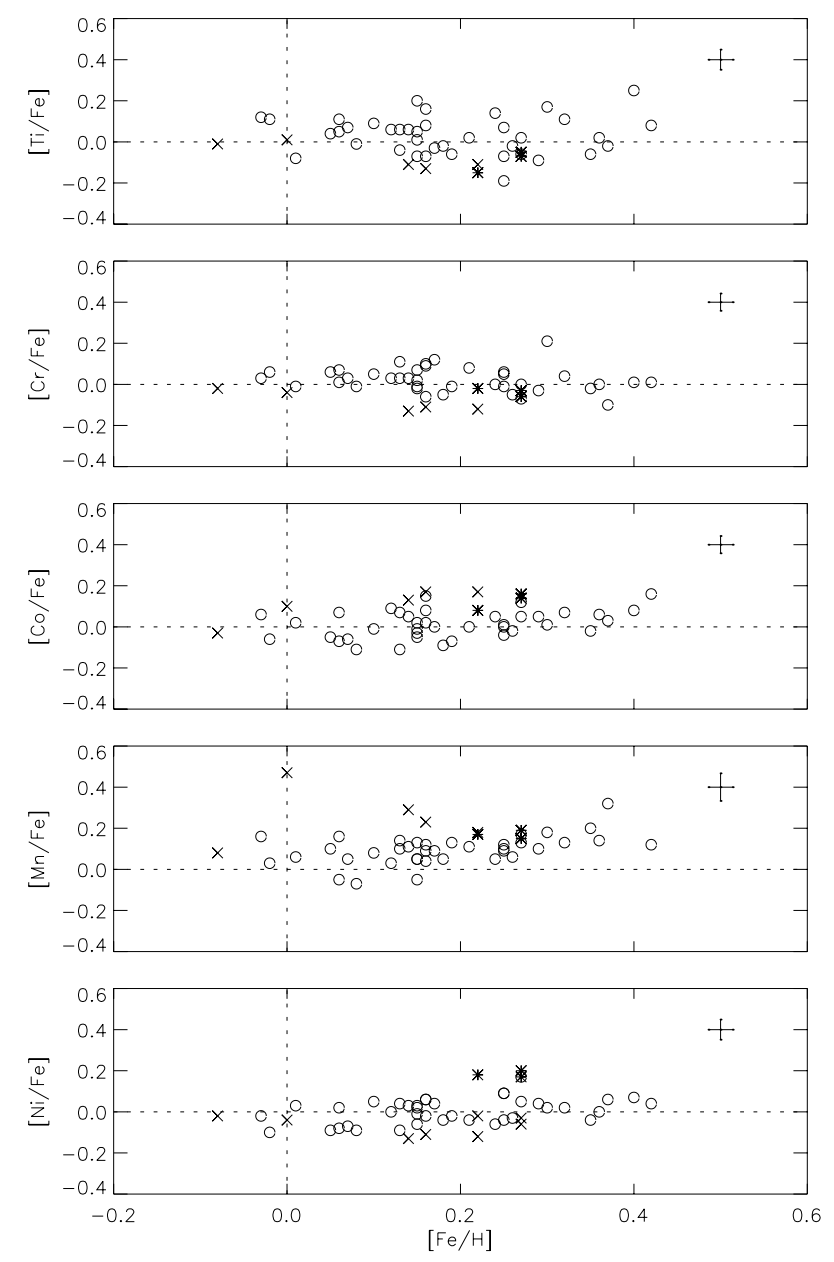

Fig. 8. Our abundances relative to Fe. $\times$ symbols denote the five $\mathrm{K}$ dwarf stars while $*$ symbols denote the stars from Barbuy \& Grenon (1990)

to be put on the same scale as the Nissen \& Edvardsson (1992) abundances.

Three of our stars, HD 37986, HD 77338 and HD 87007, have previously been studied by Barbuy \& Grenon (1990). These authors derive oxygen abundances and metallicities for a group of 11 dwarf stars. The stars were selected on the basis of their kinematics and claimed to represent the "local bulge population". The stars fell clearly above the oxygen trend expected from simple models of galactic chemical evolution (their Fig. 1). The results were interpreted as possible evidence for a rapid, and probably early, enrichment of the gas in the galactic Bulge. For most of the 11 stars in their study, Barbuy \& Grenon (1990) derived the same abundance from the forbidden line as from the triplet lines. This is not the case for the majority of the stars in our study, see Fig. 11. As shown in Table 12, for the three stars in common with Barbuy \& Grenon (1990), we find that our iron abundances are lower by 0.2 dex as compared to their results, while the oxygen abundances derived from the triplet lines stay the same relative to iron. The largest discrepancy in derived oxygen abundance is found for the forbidden line in HD 87007. Unfortunately, the error in our determination of this abundance is rather large. The spectrum is one of our poorer, with a $\mathrm{S} / \mathrm{N}$ of only $\sim 80$. The estimated error in $[\mathrm{O} / \mathrm{H}]$ from noise might then be as large as 0.5 dex. Thus, it is possible that the high abundance derived by us from the forbidden line for HD 87007 is due to errors. For the two other stars the forbidden oxygen line was, 

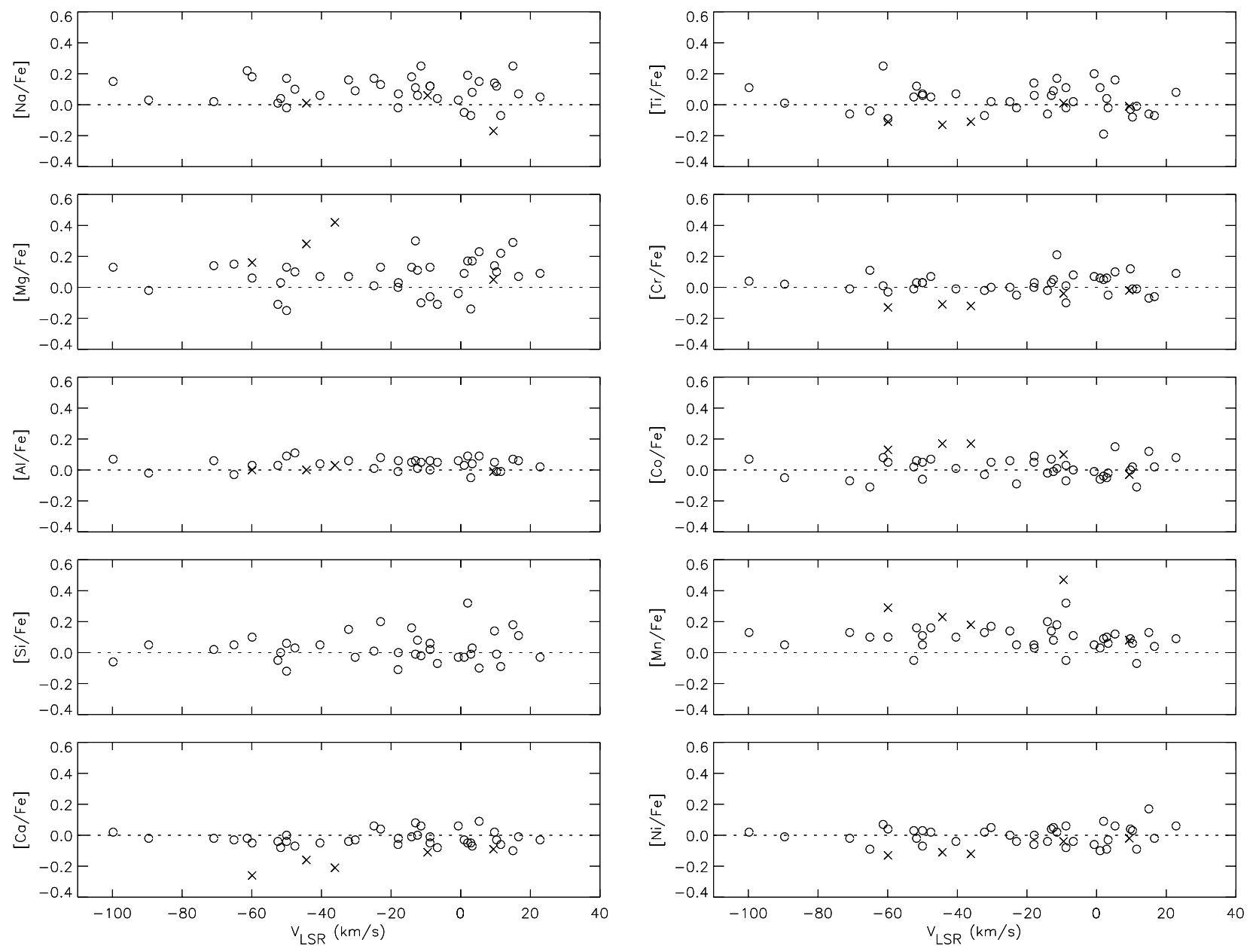

Fig. 9. Our abundances relative to $V_{\mathrm{LSR}} \times \times$ symbols denote the five $\mathrm{K}$ dwarf stars

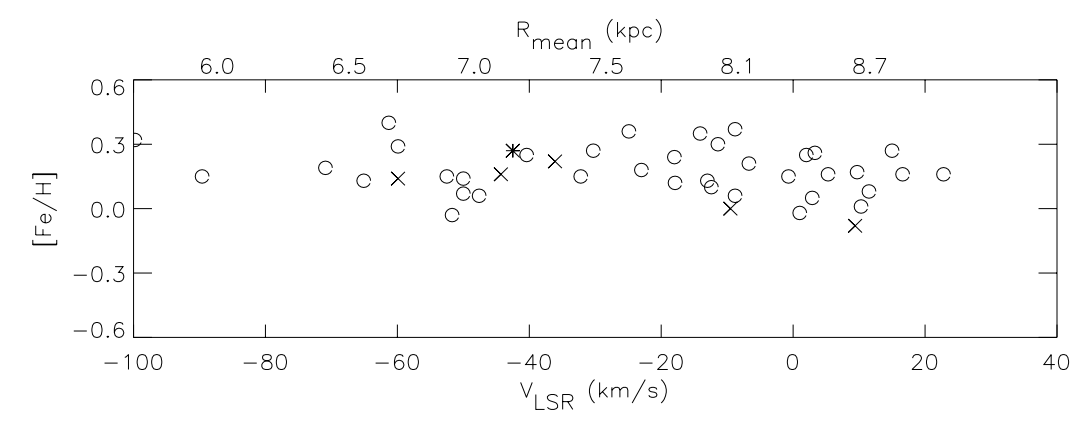

Fig. 10. Iron abundances relative hydrogen vs. $V_{\mathrm{LSR}} . \times$ signs denote the five $\mathrm{K}$ dwarf stars and HD 87007 is denoted by a $*$ sign

unfortunately, heavily obscured by telluric lines and could not be used.

We only have access to velocity data for one of the stars, HD 87007 (Barbuy, private communication). It has a $V_{\mathrm{LSR}}$ velocity of $-42.5 \mathrm{~km} \mathrm{~s}^{-1}$ which means that it would not satisfy the velocity criteria of our high-velocity sample designed to represent the inner part of the disk. The high $U_{\text {LSR }}$ velocity (radial velocity in the galactic plane of symmetry) of the star, however, gives a total spatial velocity which fulfils the requirement of membership in our high velocity sample. Castro et al. (1997) have studied 9 other high velocity stars from the work by Grenon (1989). Their results support our results. It is, however, difficult to make a clear comparison since velocity data for these stars have not been published.

In Fig. 12 we plot $[\mathrm{O} / \mathrm{Fe}]$ vs. $V_{\text {LSR }}$ for our stars as well as the, generally, more metal-poor disk stars in the sample of Nissen \& Edvardsson (1992). For the combined sample one may trace a tendency for $[\mathrm{O} / \mathrm{Fe}]$ to decrease with increasing $V_{\mathrm{LSR}}$, but this is not shown by our sample alone. 

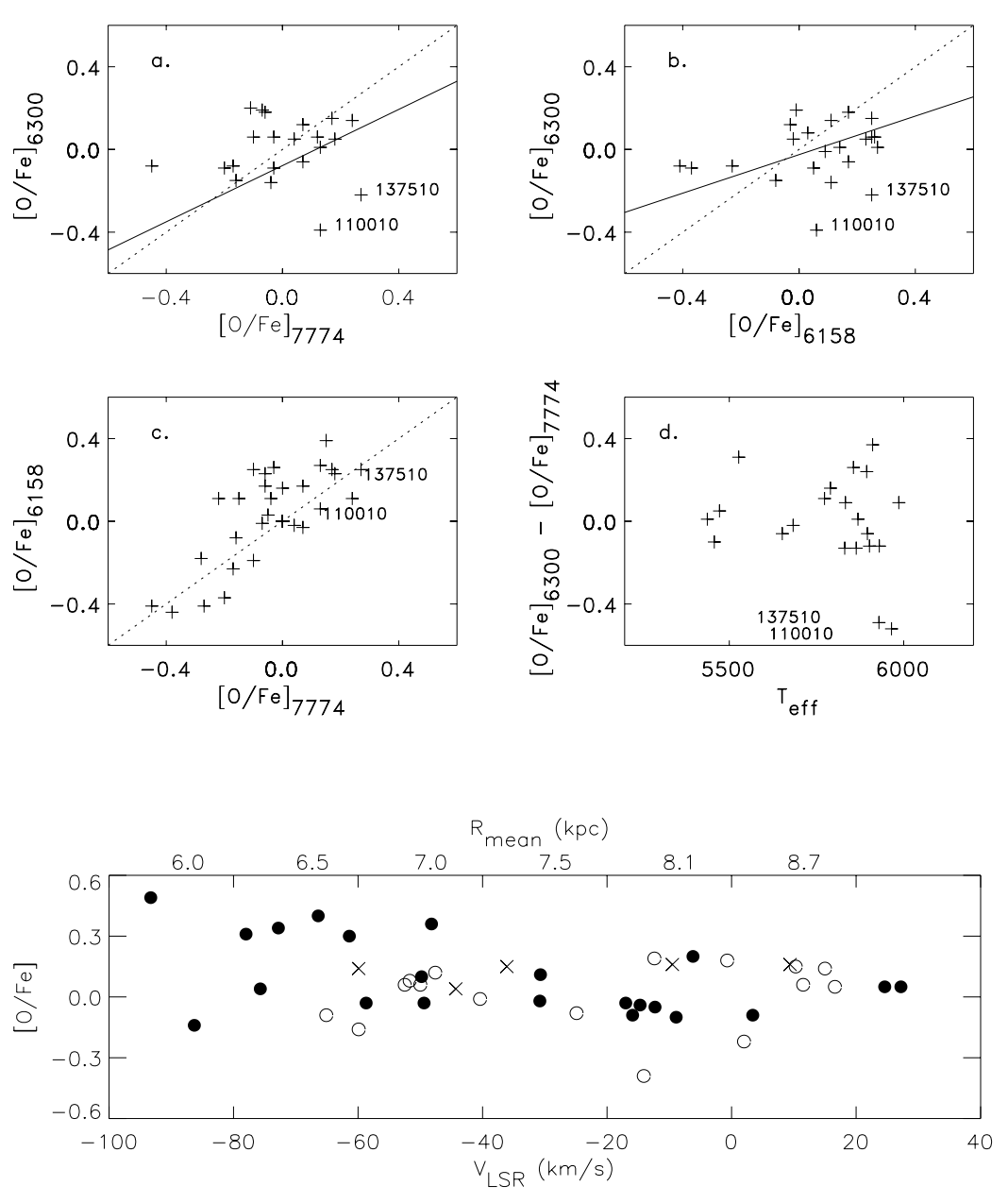

Fig. 11. Oxygen abundances derived from different abundance criteria are compared, panel a), b) and c) On each axis is the wavelength of the criterion indicated. The $7774 \AA$ oxygen abundance represents the mean of the results for the three triplet lines. The one-to-one relations are indicated by dotted lines and the relations found by Edvardsson et al. (1993a) by solid lines. In panel $\mathbf{d}$ ) we show the difference between abundances derived from $6300 \AA$ and the triplet lines as a function of effective temperature

Fig. 12. Oxygen abundances, $\circ$ and $x$ this work and $\bullet$ Nissen \& Edvardsson (1992), as function of $V_{\mathrm{LSR}}$. At the top of the figure is the mean galactocentric distance, $R_{\mathrm{m}}=\left(R_{\text {perigal. }}+R_{\text {apogal. }}\right) /$ $2 \mathrm{kpc}$, indicated for different velocities. The relation between $V_{\mathrm{LSR}}$ and $R_{\mathrm{m}}$ is taken from Fig. 1 in Edvardsson et al. (1993b). The star with the lowest oxygen to iron abundance ratio is HD 110010
From Fig. 13 we conclude that the oxygen abundance in general keeps declining relative to the iron abundance also for $[\mathrm{Fe} / \mathrm{H}]>0.1 \mathrm{dex}$. This is not inconsistent with what Nissen \& Edvardsson (1992) found in this metallicity range.

Oxygen is produced in massive stars exploding as supernovae of types II, Ib and Ic, Woosley \& Weaver (1995) and Thielemann et al. (1996). Therefore, oxygen is expected to rapidly build up at early times in the Galaxy or in any region which has experienced substantial star formation "lately". [O/Fe] starts to decline once the iron producing supernovae start contributing more significantly to the enrichment of the interstellar gas. This decline starts at $[\mathrm{Fe} / \mathrm{H}] \sim-1$ dex in the galactic disk, or at even lower metallicities.

In Fig. 13 we compare our data with three different theoretical models of the galactic chemical evolution. The model by Matteucci \& François (1989) clearly shows the envisaged decline in $[\mathrm{O} / \mathrm{Fe}]$ after $[\mathrm{Fe} / \mathrm{H}]=-1.0$ dex, i.e. when supernovae type Ia start to contribute to the enrichment of the gas. This can be compared with a more recent model, taking the effects of metallicity dependent supernova yields into account, by Prantzos \& Aubert (1995).
The difference between the two models by Prantzos \& Aubert (1995) is not large for lower metallicities but from around solar metallicity there is an increasing discrepancy between their two models. If this suggested trend continues to higher $[\mathrm{Fe} / \mathrm{H}]$ the model using metallicity dependent yields would be favoured by our data. The model by Pagel \& Tautvaišienè (1995) is a simple analytic model, which assumes supernovae type Ia to give their yields after a fixed time delay. In spite of its simplicity it fits the data remarkably well and may suggest that the basic understanding of the processes involved is correct.

Tsujimoto et al. (1995) studied the abundance gradients in the galactic disk by means of a viscous disk model of galactic chemical evolution. As can be seen in their Fig. 8 differences in oxygen abundances as a function of radial distance from the galactic centre are predicted to be so small that it would hardly be possible to resolve this in a study as ours where such a small part of the radius is spanned. This is compatible with our results in Fig. 13. We note that their model predicts (their Figs. 7 and 8) $[\mathrm{O} / \mathrm{Fe}]$ vs. $[\mathrm{Fe} / \mathrm{H}]$ to flatten out at solar metallicities. If this suggested trend is continued when the models are 
Table 12. Stellar parameters and oxygen abundances for the stars in common between this work and that by Barbuy \& Grenon (1990). For each star we give our iron abundandance as derived from spectral lines, $[\mathrm{Fe} / \mathrm{H}]$, the metallicity as quoted by Barbuy \& Grenon, $[\mathrm{M} / \mathrm{H}]$ and the oxygen abundances derived from the different criteria as indicated. For each criterion we give our resluts on the first line and the results by Barbuy \& Grenon on the second. $[\mathrm{O} / \mathrm{Fe}]_{6158}$ was not measured by them

\begin{tabular}{lrrr}
\hline ID & HD 37986 & HD 77338 & HD 87007 \\
\hline$[\mathrm{Fe} / \mathrm{H}]$ & 0.27 & 0.22 & 0.27 \\
{$[\mathrm{M} / \mathrm{H}]$} & 0.47 & 0.45 & 0.43 \\
\hline$[\mathrm{O} / \mathrm{Fe}]_{6300}$ & & & 0.54 \\
& 0.15 & 0.20 & 0.00 \\
{$[\mathrm{O} / \mathrm{Fe}]_{7770}$} & 0.15 & 0.29 & 0.27 \\
& 0.23 & 0.20 & 0.20 \\
{$[\mathrm{O} / \mathrm{Fe}]_{6158}$} & 0.39 & -0.04 & 0.27 \\
\hline
\end{tabular}

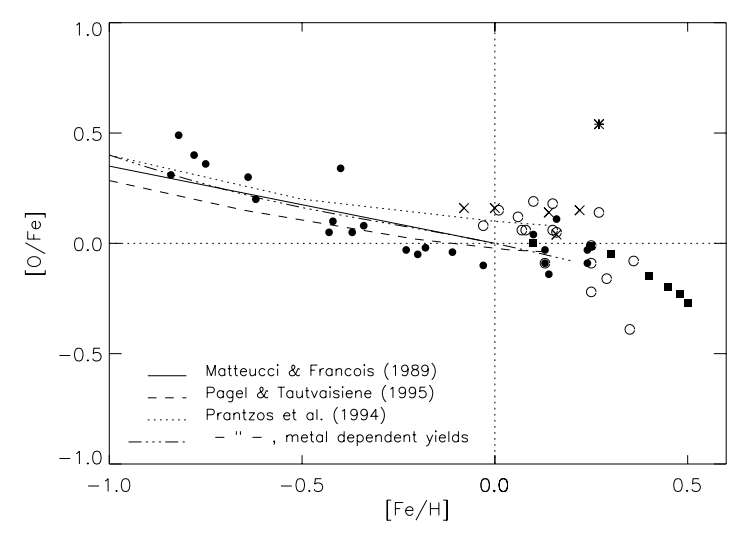

Fig. 13. Oxygen abundances from this work, $\circ$ symbols, and from Nissen \& Edvardsson (1992), • symbols. $\times$ symbols denote our five $\mathrm{K}$ dwarf stars, * symbol HD 87007 and the filled boxes data from Castro et al. (1997). The star with lowest $[\mathrm{O} / \mathrm{Fe}]$ is HD 110010

further evolved, it would be difficult to reconcile them with our data, and with the results by Castro et al. (1997).

\subsection{Sodium}

One of the aims with our study was to determine whether or not the upturn of $[\mathrm{Na} / \mathrm{Fe}]$ vs. $[\mathrm{Fe} / \mathrm{H}]$ found by Edvardsson et al. (1993a) is real and if so, if it is an effect of a mixture of stars from different populations, $\mathrm{cf}$ Figs. 8, 9, 14 and 15. We confirm that the upturn is real. However, the upturn is less steep in our study than what one could trace from the scattered diagram of Edvardsson et al. (1993a).

In Fig. 15 we have divided our sample into stars representing the disk interior to the solar orbit and the solar orbit and made linear least-square fits to the data. In panel a. we show all 47 stars and a least-square fit with the $\mathrm{K}$ dwarf stars and the stars from Barbuy \& Grenon (1990) excluded. In panel b. we show the stars that represent the disk interior to the solar orbit. There is no appreciable difference found between this sample and the whole sample. In panel c. and $\mathrm{d}$. we have defined the solar orbit sample in two different ways. In c. it contains all stars with $V_{\mathrm{LSR}}<45 \mathrm{~km} \mathrm{~s}^{-1}$ and in d. with $V_{\mathrm{LSR}}<30 \mathrm{~km} \mathrm{~s}^{-1}$. The sample in panel c. also has a behaviour indistinguible from that of panel a. However, the $V_{\mathrm{LSR}}<30 \mathrm{~km} \mathrm{~s}^{-1}$ seem to show a somewhat steeper trend.

An interesting question is now what difference in $[\mathrm{Na} / \mathrm{Fe}]$ one would have reason to expect for stars formed at different $R_{\mathrm{m}}$. Using the data of Edvardsson et al. (1993a) we estimate that the minimum value of $\mathrm{d}[\mathrm{Na} / \mathrm{Fe}] / \mathrm{d} R_{\mathrm{m}}$ is about $0.05 \mathrm{dex} / \mathrm{kpc}$. Extrapolating this to the metal-rich stars and to $R_{\mathrm{m}} \sim 6 \mathrm{kpc}$ one finds at the most a difference of 0.1 dex between our two samples. Implicit in this assumption is then that the population of metal-rich stars at $6 \mathrm{kpc}$, which is very sparsely represented in the sample of Edvardsson et al. (1993a), is not qualitatively different from that in the solar neighbourhood. The results obtained in the present study supports this and indicate that the difference in $[\mathrm{Na} / \mathrm{Fe}]$ is, in fact, at the most 0.05 dex.

In this connection, one should also note that orbital diffusion may well mask the possible differences between stars formed at $6 \mathrm{kpc}$ and $8 \mathrm{kpc}$. E.g., stars of solar age in an orbit with $R_{\mathrm{m}}=8.5 \mathrm{kpc}$ may have migrated from an orbit with $R_{\mathrm{m}}=6-7 \mathrm{kpc}$ (cf. Wielen et al. 1996). The mixture of stars with different original orbits, in combination with a radial galactic gradient of $[\mathrm{Fe} / \mathrm{H}]$, was proposed by Wielen et al. (1996) to explain the unexpectedly high scatter in $[\mathrm{Fe} / \mathrm{H}]$ of 0.2 dex for solar type stars, with similar age and similar present $R_{\mathrm{m}}$, found by Edvardsson et al. (1993a). From the work of Wielen et al. (1996) we estimate that two samples of stars with $\left\langle R_{\mathrm{m}}\right\rangle=8.5$ and $6.5 \mathrm{kpc}$, respectively, would then be mixed by orbital diffusion so much that the population effects in abundances only would show up to about half the expected size as compared with the situation if orbital diffusion is not present. Although the reason for the great inhomogeneities in the gravitational potential, needed to account for the orbital diffusion of this magnitude, is not known, we conclude that the effects looked for by dividing the total sample of stars according to the velocity criteria used here, might be diminished considerably by this phenomenon.

\subsection{Aluminium}

Aluminium is produced in heavy single stars, with $M>$ $8 M_{\odot}$. The scatter, as well as the internal line-to-line scatter, in our data is considerably smaller than in Edvardsson et al. (1993a). The upturn in $[\mathrm{Al} / \mathrm{Fe}]$ vs. $[\mathrm{Fe} / \mathrm{H}]$ indicated in their data is not obviously present in ours, see Fig. 14. 

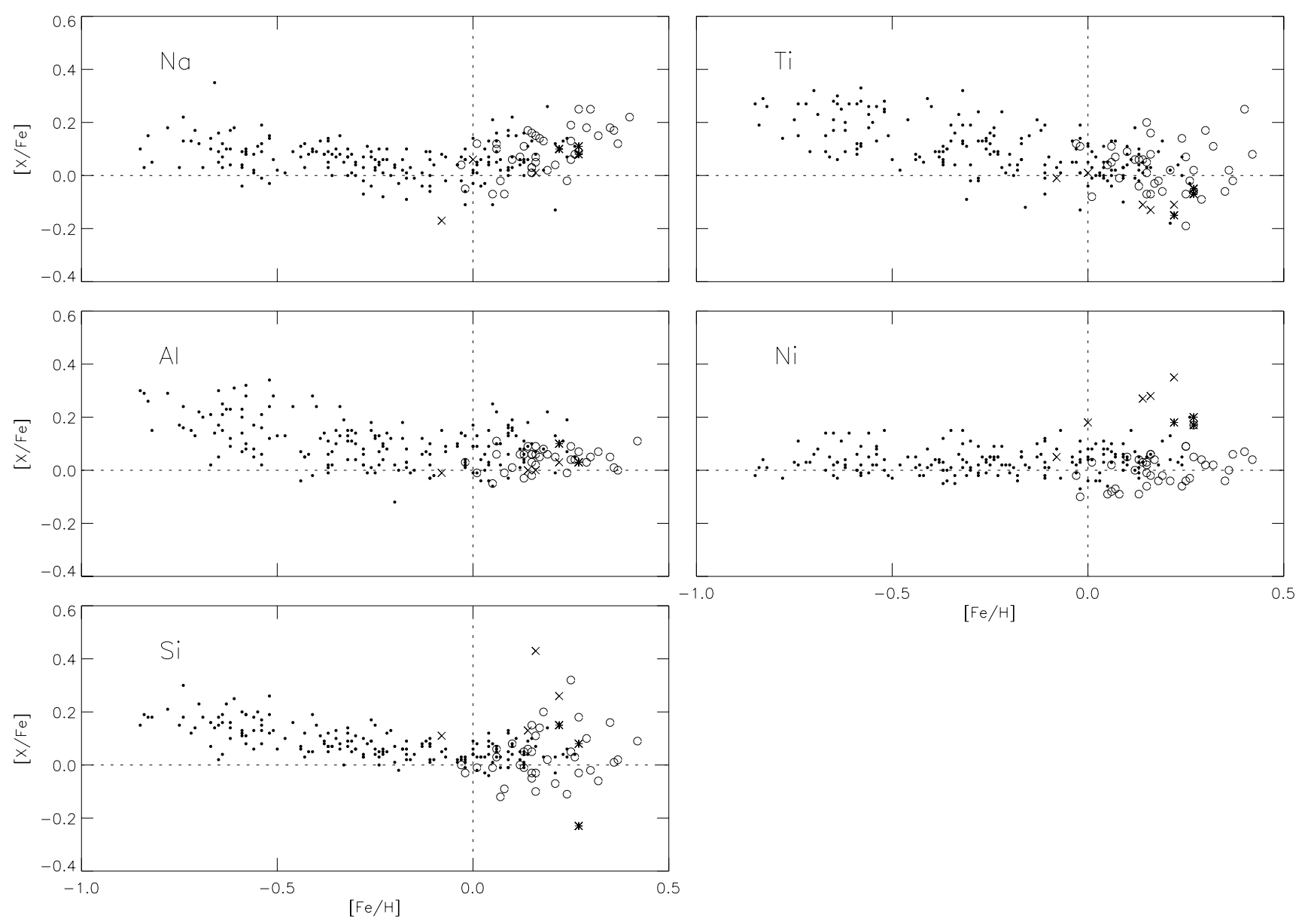

Fig. 14. Abundances from this study compared with results from Edvardsson et al. (1993a). On the vertical axes we give [X/Fe] where $\mathrm{X}$ is the element indicated in the upper left corner of each panel. o symbols denote our results except the five $\mathrm{K}$ dwarf stars which are denoted by $\times$ symbols and the three stars from Barbuy \& Grenon (1990) which are denoted by $*$ symbols. Results from Edvardsson et al. are denoted by $\bullet$ symbols
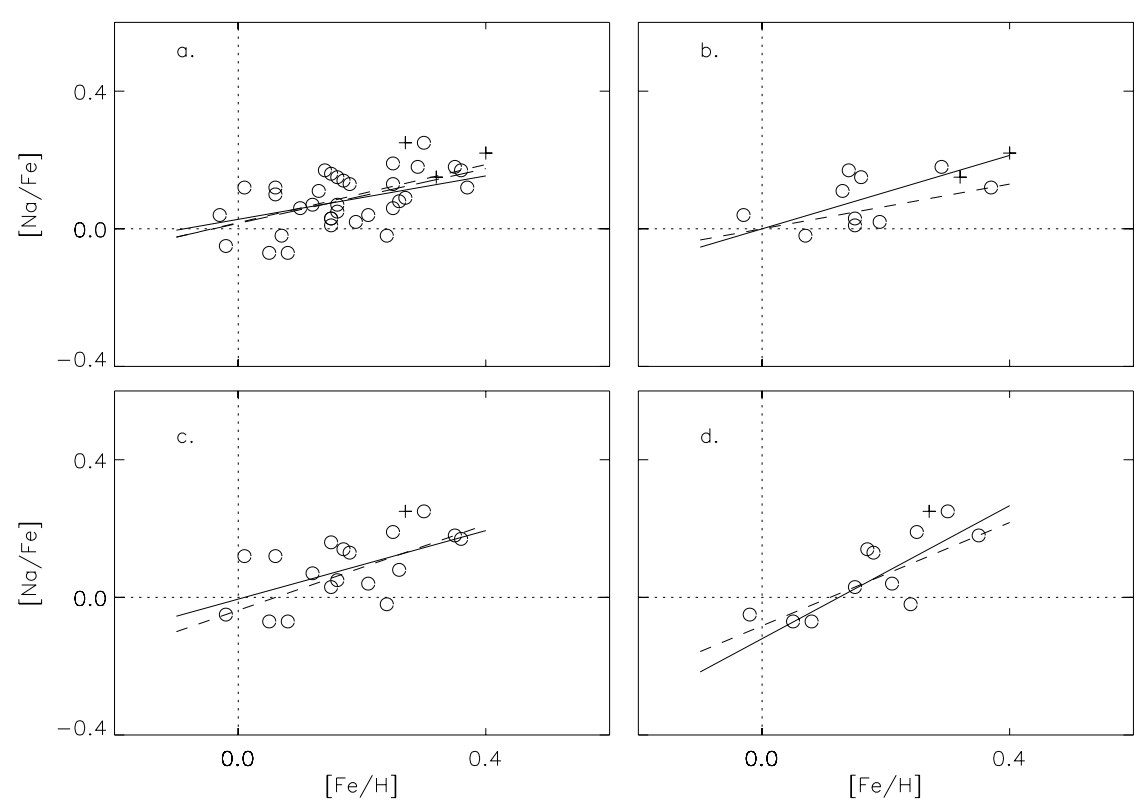

Fig. 15. In this figure we explore the different velocity samples as indicators of differences in galactic chemical evolution in adjacent parts of the galactic disk. The five $\mathrm{K}$ dwarf stars and the stars from Barbuy \& Grenon (1990) are excluded from this discussion. In panel a) we show $[\mathrm{Na} / \mathrm{Fe}]$ for all stars. Error-bars are smaller than the symbols. In panel b) are the stars with $V_{\mathrm{LSR}}<-50 \mathrm{~km} \mathrm{~s}^{-1}$ and/or $Q_{\mathrm{LSR}}>$ $60 \mathrm{~km} \mathrm{~s}^{-1}$ shown. In panel c) stars with $Q_{\mathrm{LSR}}<45 \mathrm{~km} \mathrm{~s}^{-1}$ and in panel d) stars with $Q_{\mathrm{LSR}}<30 \mathrm{~km} \mathrm{~s}^{-1}$. Solid lines show the result of a weighted linear least square fit to the data and the dashed lines show least distance fits to the same data. Stars denotes by + symbols have sodium abundance determined from one line only, and are not used in the error weighted fits 


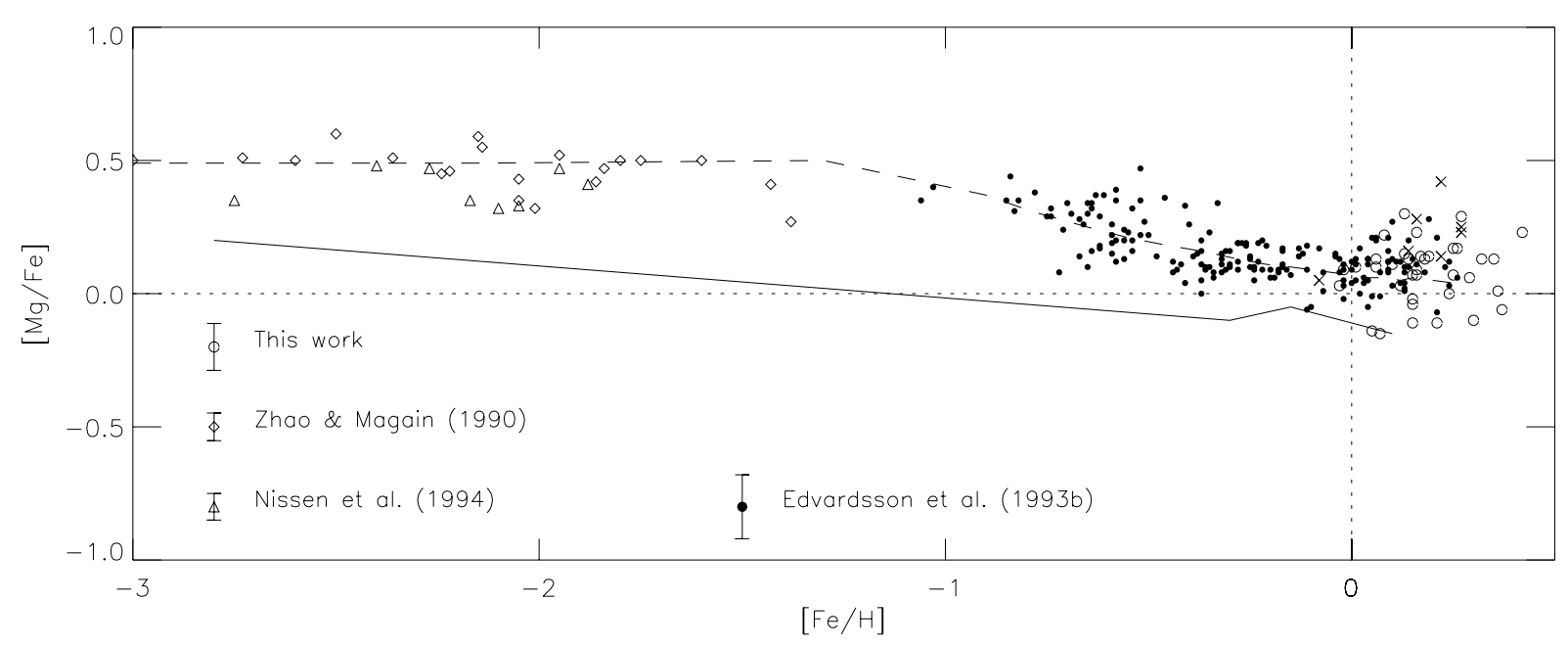

Fig. 16. Magnesium vs. iron abundances from several studies, as given in the figure, as well as model calculations of the galactic chemical evolution from Pagel \& Tautvaišienè (1995) (dashed line) and Timmes et al. (1995) (solid line). $\times$ signs denote the K dwarf stars in our sample. Indicated error bars refer to the error in the mean

The two studies use different lines for the abundance analysis. The small scatter in $[\mathrm{Al} / \mathrm{Fe}]$ for $[\mathrm{Fe} / \mathrm{H}]>0.0$ dex is also evident in Morell (1994). The lines used by Morell (1994) and us, 6696.03 $\AA$ and 6698.66 $\AA$, are situated in a part of the stellar spectrum which is clean. Thus, we expect no problems with continuum fitting and blends and errors arising from measurements of the line strengths should be negligible. The trend in Fig. 14 and its similarity with, e.g., that of $\mathrm{Ca}$ (Fig. 18) suggests a similar origin in core-collapse supernovae.

\subsection{Magnesium}

Due to the large line-to-line scatter in our magnesium data it is not possible to determine here if the large scatter found by Edvardsson et et al. (1993a) is real or not. We note, however, that we get roughly the same amount of scatter as they do, Fig. 16. There is no evidence in our data for a correlation between kinematics and $[\mathrm{Mg} / \mathrm{Fe}]$ ratios of the stars, Fig. 9.

Magnesium is, like oxygen, usually assumed to be formed only in core-collapse supernovae through hydrostatic carbon burning. Timmes et al. (1995) are not so successful in describing the over-all evolution of magnesium abundances in the Galaxy. This may suggest that the production of $\mathrm{Mg}$ is not fully understood at present. The simple-minded model with the delayed yield formalism is more successful in this respect, Pagel \& Tautvaišienė (1995).

\subsection{Silicon}

We only use two lines to determine silicon abundances while Edvardsson et al. (1993a) used eight lines. We find a much larger scatter in our data than they do, Fig. 14.
It has not been possible, from our data, to determine the origin of neither the line-to-line scatter nor the star-tostar scatter. When inspecting the lines one by one no line seems to stand out in terms of derived abundances. Nor do we find any signs of the scatter to be an effect of different stellar populations mixing, see e.g. Fig. 9.

\subsection{Calcium}

Comparison of our calcium abundances with those of Edvardsson et al. (1993a), in Fig. 17, confirms their finding that the $[\mathrm{Ca} / \mathrm{Fe}]$ flattens out towards higher metallicities. Also, there is no obvious difference between stars with different galacto-centric mean distances, Fig. 9.

As is evident from Figs. 8 and 18 the K dwarf stars exhibit a behaviour which is very different from the rest of the sample. They seem to have a mean calcium relative to iron abundance $\sim 0.2$ dex lower than the mean abundance for the rest of the stars. When plotting $[\mathrm{Ca} / \mathrm{Fe}]$ as a function of effective temperature we see that the stars with low $\mathrm{Ca}$ abundances have the lowest effective temperatures, cf. Fig. 18.

In a high resolution, high signal-to-noise abundance study of dwarfs and giants in the disk by Abia et al. (1988), there are six dwarf stars in the same metal and effective temperature range as our $\mathrm{K}$ dwarf stars. In Fig. 4a in Abia et al. (1988) the same features as in Fig. 18 can be seen, i.e. cool, metal-rich stars show up as underabundant in calcium. In another study, Gratton \& Sneden (1987), of light elements in field disk and halo stars we also find support for such a behaviour of calcium in cool dwarf stars.

The key to these low $[\mathrm{Ca} / \mathrm{Fe}]$ measures may lie in overionization. Drake (1991) performed non-LTE calculations 


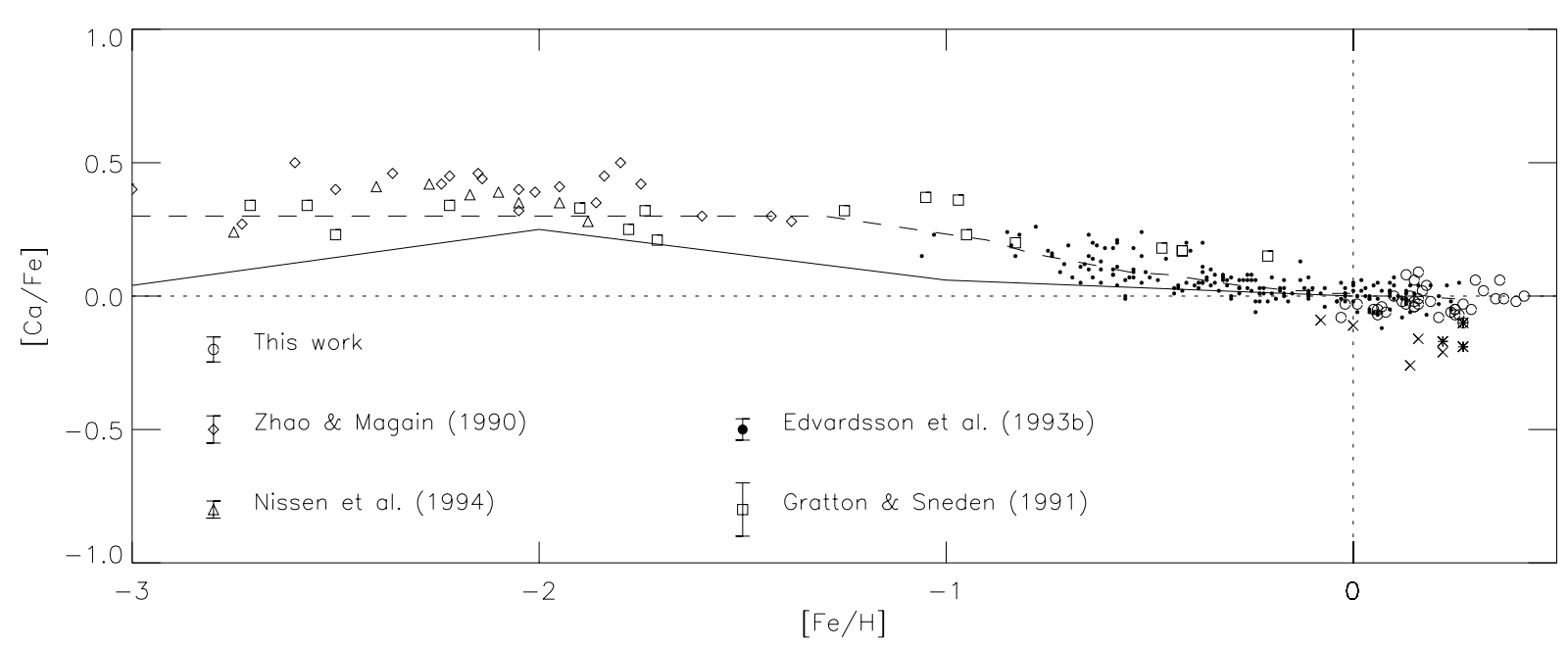

Fig. 17. Calcium abundances from several studies, as given in the figure, as well as model calculations of the galactic chemical evolution from Pagel \& Tautvaišienè (1995) (dashed line) and Timmes et al. (1995) (solid line). Typical error bars are indicated for each study. $\times$ symbols denote $\mathrm{K}$ dwarf stars and $*$ the stars in common with Barbuy \& Grenon (1990)
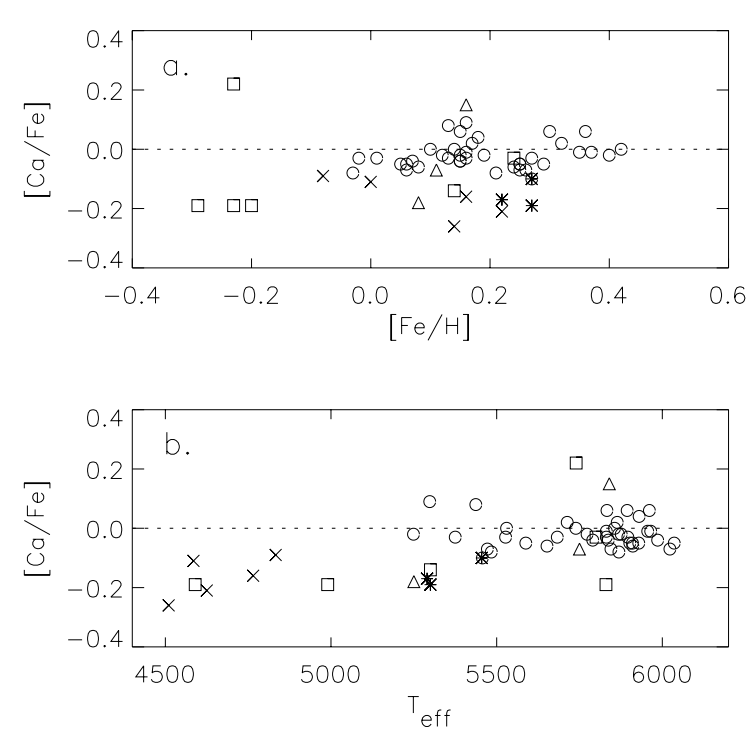

Fig. 18. Calcium abundances for our stars, except the K dwarf stars, o symbols. The $\mathrm{K}$ dwarf stars are denoted by $\times$ symbols. Abundances for solar-type stars from Abia et al. (1988), $\square$ symbols, and Gratton \& Sneden (1987), $\triangle$ symbols, and the stars from Barbuy \& Grenon (1990), * symbols

for calcium for a range of stellar parameters. He showed that the difference between an abundance derived under non-LTE and LTE conditions varies strongly with effective temperature and surface gravity, and less strongly with metallicity. Drake (1991) finds that the non-LTE effect on abundances of $\mathrm{Ca}$ in $\mathrm{G}$ and $\mathrm{K}$ dwarf stars increase considerably with decreasing effective temperature. From his Figs. 4, 7 and 8, we estimate the correction factor for weak lines in a dwarf star of at least solar metallicity with an effective temperature of $4500 \mathrm{~K}$ to be on the order of 0.3 dex. Such an adjustment would indeed put the $\mathrm{K}$ dwarf stars right on the line, $[\mathrm{Ca} / \mathrm{Fe}]=0.0$ dex. This suggests that the calcium abundance may vary in lockstep with the iron abundance also for metal-rich $\mathrm{K}$ dwarf stars.

\subsection{Titanium}

[Ti/Fe] was shown by Edvardsson et al. (1993a) to be a slowly decreasing function of $[\mathrm{Fe} / \mathrm{H}]$. The decline may continue also for higher iron abundances. We use $10-12$ lines to derive titanium abundances for our stars, while Edvardsson et al. (1993a) used four. In spite of our, presumably, smaller random errors in the abundance determination for each star, as is shown in Fig. 14 we still find the same and comparatively large scatter in the abundances found by Edvardsson et al. (1993a).

Inspection of derived stellar abundances as a function of excitation energy for the lower level in the transition for each line indicated no presence of non-LTE effects or blends. However, also for titanium we found no evidence that the large star-to-star scatter should be a result of a mixing of stars with different mean-perigalactic distances, i.e. different $V_{\mathrm{LSR}}$ velocities, see Fig. 9.

\subsection{Scandium and Vanadium}

Abundances derived from Sc I lines are unreliable and we therefore only present abundances determined from Sc II lines, Fig. 19. Scandium exhibits some scatter but seems to vary in lockstep with iron.

For vanadium the atom is represented by two lines and the ion by one. We present the data derived from lines of 
the atom. Also vanadium appears to vary in lockstep with iron over the metallicity range studied.
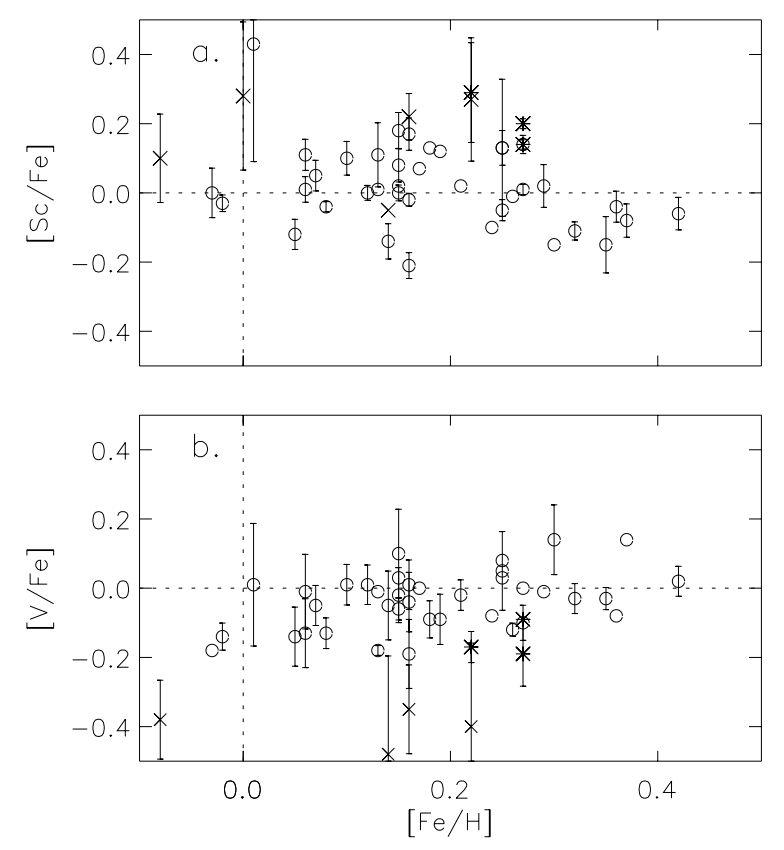

Fig. 19. Scandium abundances from Sc II and vanadium abundances from V I. Error bars indicate the error in the mean. Stars with no error bar means that the abundance was derived from a single line. $\times$ symbols denote $\mathrm{K}$ dwarf stars and * symbols the stars from Barbuy \& Grenon (1990)

\subsection{Chromium}

For the most metal-rich stars chromium, as well as other iron peak elements, varies in lockstep with iron, Fig. 8. The overall evolution of $[\mathrm{Cr} / \mathrm{Fe}]$ seems to be well described by Timmes et al. (1995) using the supernova yields by Woosley \& Weaver (1995), Fig. 20. The flatness of the relation between $[\mathrm{Cr} / \mathrm{Fe}]$ and $[\mathrm{Fe} / \mathrm{H}]$ can be understood as a consequence of that massive stars with solar initial metallicities produce enough chromium to balance the iron production by SNIa, cf. Timmes et al. (1995).

\subsection{Manganese and Cobalt}

Few studies of stellar abundances have been made for these elements. Manganese abundances were measured by Gratton (1989) for 25 metal-poor giants and dwarfs. Cobalt abundances were obtained by Gratton \& Sneden (1991) for 17 metal-poor (mostly) giant and dwarf stars and by Ryan et al. (1991) for 19 dwarf and giant stars.

Five lines of the manganese atom were used for determination of abundances, three weak and two stronger lines. $[\mathrm{Mn} / \mathrm{Fe}]$ scales with $[\mathrm{Fe} / \mathrm{H}]$, but with a tendency to increase for $[\mathrm{Fe} / \mathrm{H}]>-1.0$ dex, Fig. 21. This increase seems to continue beyond $[\mathrm{Fe} / \mathrm{H}]>0.0$ dex. We use seven lines arising from the atom to determine cobalt abundances. From our data cobalt seems to vary in lock-step with iron for $0.0 \mathrm{dex}<[\mathrm{Fe} / \mathrm{H}]<0.3$ dex.

Manganese and cobalt belong to the group of iron-peak elements. These elements are thought to be formed during explosive silicon burning in supernova explosions and nuclear statistical equilibrium (Woosley \& Weaver 1995).

Timmes et al. (1995) find that the rise in $[\mathrm{Mn} / \mathrm{Fe}]$ vs. $[\mathrm{Fe} / \mathrm{H}]$ from $[\mathrm{Fe} / \mathrm{H}] \sim-1$ dex is due to the over-production of manganese in supernovae type Ia and in heavy stars with solar metallicity, as compared with the iron production, while for cobalt the production of iron in supernovae type Ia is balanced by production of cobalt in supernovae resulting from massive stars with initially solar metallicity.

\subsection{Nickel}

We have used 12 lines from the nickel atom to obtain abundances; Edvardsson et al. (1993a) used 20 lines. Like Edvardsson et al. (1993a) we find that nickel varies in lockstep with iron, and this continues also for higher metallicities, Fig. 14. For the stars in common between the studies the nickel abundances derived are in excellent agreement, although our study does not show the slight offset found by Edvardsson et al. (1993a).

Here, we also note an interesting behaviour of the $\mathrm{K}$ dwarf stars, namely that they show larger nickel abundances than the rest of the sample. The large number of lines together with the fact that the lower excitation energies for the lines span a range of values $(1.9-5.3 \mathrm{eV})$ and that the formal error for each star is small makes departures from excitation equilibrium an unlikely explanation for this effect.

Overionization, for most stars but less for the cool ones, or blends are possible but neither very probable explanations. The phenomenon needs further systematic study.

\subsection{3. $r$ - and s-process elements}

Most of the heavy elements $(A>70)$ are formed through the $r$ - and $s$-processes. For some of them one of the processes contributes much more than the other. The $s$ process contributes most, for the solar system composition, to $\mathrm{Y}(73 \%)$ and $\mathrm{Zr}(79 \%)$ while $\mathrm{Eu}$ is to $90 \%$ formed in the $r$-process, according to Anders \& Grevesse (1989). $\mathrm{Eu}$ is one of the few $r$-process elements with clean lines observable in the visual part of stellar spectra. Therefore, it is well suited for studies of the sites for the $r$-process. The relative abundances of $s$-elements produced in thermally pulsing asymptotic giant branch stars are set by the degree of the exposure to neutrons. Heavier neutron flux enhances the abundances of the heavier elements ( $\mathrm{Ba}, \mathrm{Nd}, \mathrm{Hf})$ relative to the lighter ones (Y, Zr, Mo). Molybdenum is formed 


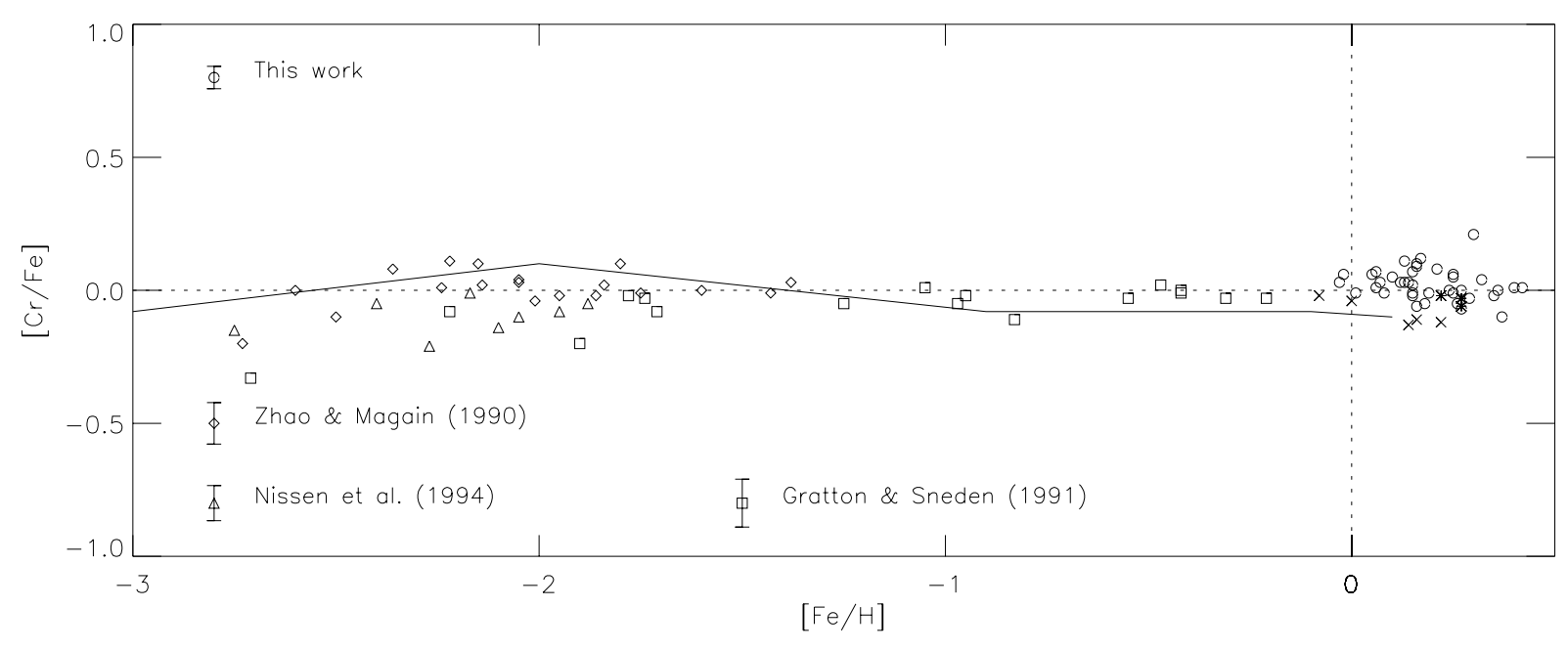

Fig. 20. Chromium data from several sources, as given in the figure, showing the galactic chemical evolution of chromium. The solid line is from Timmes et al. (1995). The chromium rich star in our sample is HD 87646. The error bars indicate the error in the mean. $\times$ symbols denote our K dwarf stars and $*$ the stars in common with Barbuy \& Grenon (1990). Chromium was not studied by Edvardsson et al. (1993a)
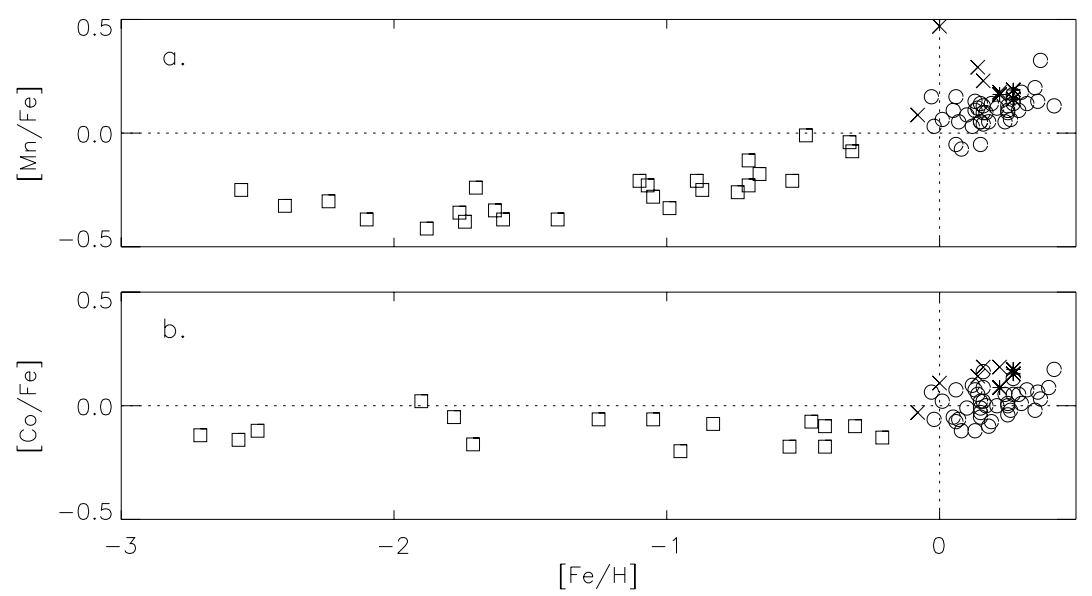

Fig. 21. Manganese, panel a), and cobalt, panel b), abundances from this work, $\circ, \times$, and $*$ symbols, and from Gratton (1989) (manganese) and Gratton \& Sneden (1991) (cobalt), $\square$ symbols

by a mixture of processes ( $p$ -,$r$ - and $s$-processes), see Anders \& Grevesse (1989).

From this knowledge one would expect the $r$-process elements to have high abundances in old stars and show a declining trend when compared to iron. This is indeed seen for europium, see figures and discussions in Mathews et al. (1992) and Woolf et al. (1995). For the $s$-process elements, on the other hand, one would expect old stars to have low $s$-element abundances while the more recently formed stars would show an increase in their $s$-element abundance, due to the long time scales for the evolution of the $s$-process sites. Such a tendency was also traced by Edvardsson et al. (1993a).

\section{Results}

Our stellar spectra contain few lines of these heavy elements that are accessible in the visual and may be securely used as abundance criteria.
We have derived abundances for a number of $s$ - and $r$ process elements, using a small number of Y II lines of suitable strength, one line each for Zr I and Mo I, two for La II and one each for NdII, EuII and HfII. We find that the metal-rich stars have roughly solar abundances of these elements relative to iron, however, with some possible departing trends.

Up to $[\mathrm{Fe} / \mathrm{H}] \sim 0.2$ dex we confirm the result by Edvardsson et al. (1993a) that $\mathrm{Y}$ varies in lock-step with iron. For the more metal-rich stars, however, there may be a decline in $[\mathrm{Y} / \mathrm{Fe}]$ (see Fig. 22). The results may, however, be due to possible effects of overionization in yttrium.

As is clear from Fig. 22 that for $\mathrm{Zr}$ there is probably a systematic trend with effective temperature, resulting in a large scatter (or even a division of stars into groups) and unreliable abundances. Blends may may also contribute to this scatter. The results for our stars do generally indicate lower $\mathrm{Nd}$ abundances than those of Edvardsson et al. 

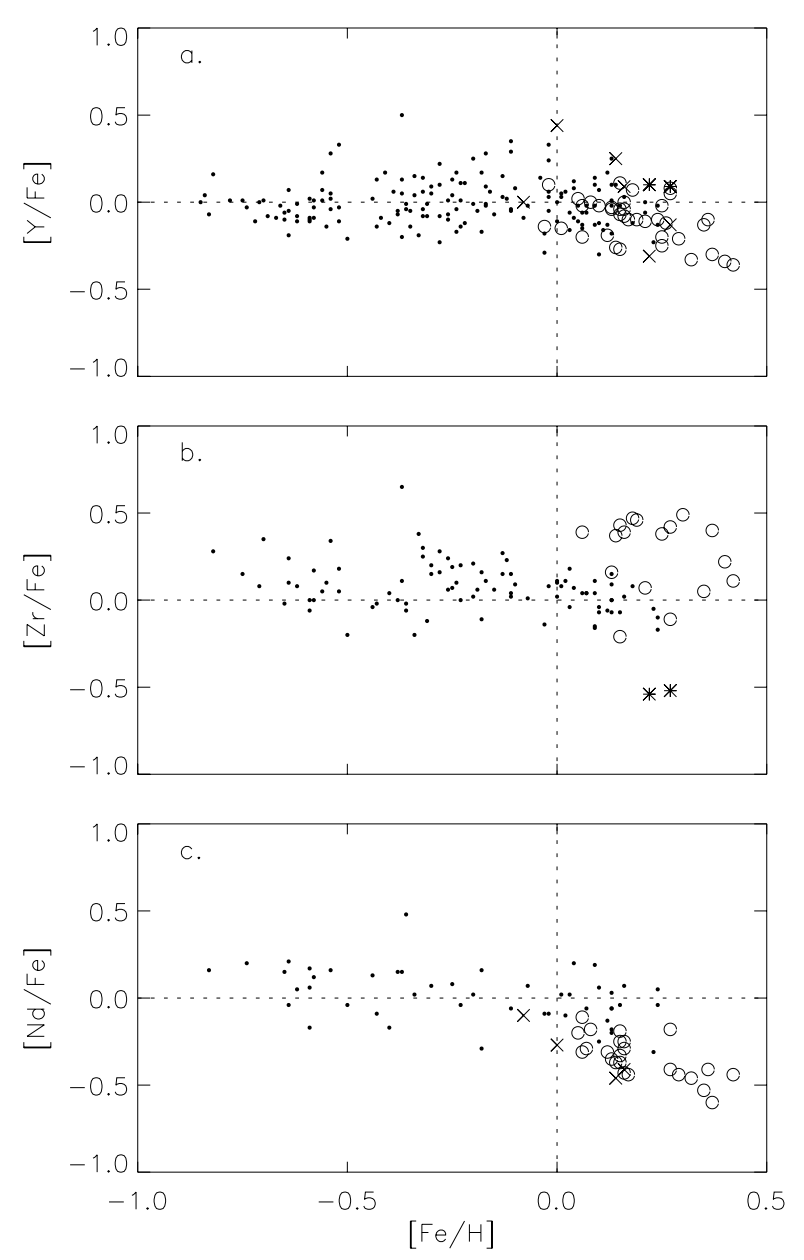

Fig. 22. Comparison of data from this study and from Edvardsson et al. (1993a) for three elements, yttrium, zirconium and neodymium. $\circ$ symbols denote our abundances, $\times$ symbols denote the $\mathrm{K}$ dwarf stars, * symbols the stars from Barbuy \& Grenon (1990) and • symbols denote abundances from Edvardsson et al. (1993a)

(1993a) and again a decrease of $[\mathrm{Nd} / \mathrm{Fe}]$ with increasing $[\mathrm{Fe} / \mathrm{H}]$.

For molybdenum, lanthanum and hafnium we have no studies to compare with and can therefore not say very much about the general evolution. The molybdenum abundances are derived from one Mo I line. Mo and Hf, and probably also La, however, show the familiar pattern, ascribed to overionization in the $\mathrm{K}$ dwarf stars.

In conclusion, the abundances of Mo, La, and Hf seem to roughly vary in lock-step with $\mathrm{Fe}$, however, with some indications that the abundance ratios decrease with increasing $[\mathrm{Fe} / \mathrm{H}]$.

In order to improve the statistics we have derived two quantities, $<s-\operatorname{light} / \mathrm{Fe}>=0.5 \cdot[\mathrm{Y} / \mathrm{Fe}]+0.3 \cdot[\mathrm{Zr} / \mathrm{Fe}]+$ $0.1 \cdot[\mathrm{Nd} / \mathrm{Fe}]$, and $<s-$ heavy $/ \mathrm{Fe}>=0.25 \cdot[\mathrm{Mo} / \mathrm{Fe}]+$ $0.5 \cdot[\mathrm{La} / \mathrm{Fe}]+0.25 \cdot[\mathrm{Hf} / \mathrm{Fe}]$. The weights in these expressions reflect the number of spectral lines measured
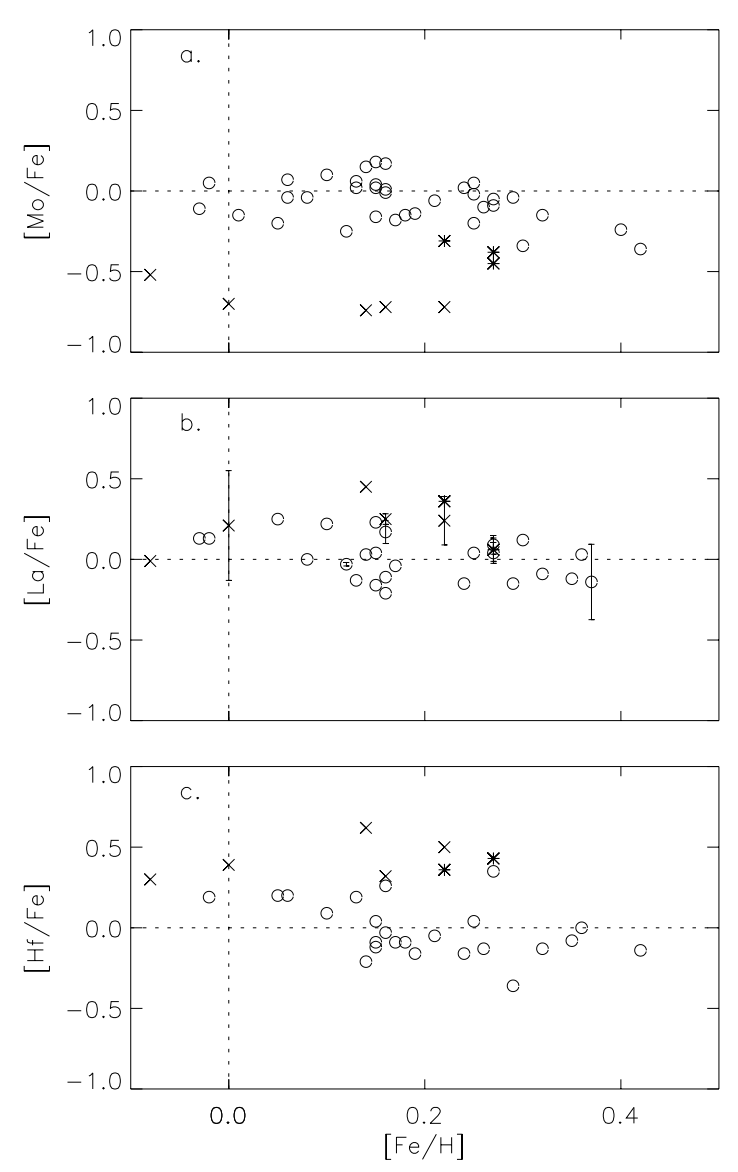

Fig. 23. Molybdium, lanthanum and hafnium abundances relative to iron. $\times$ symbols denote $\mathrm{K}$ dwarf stars and $*$ symbols the stars from Barbuy \& Grenon (1990)

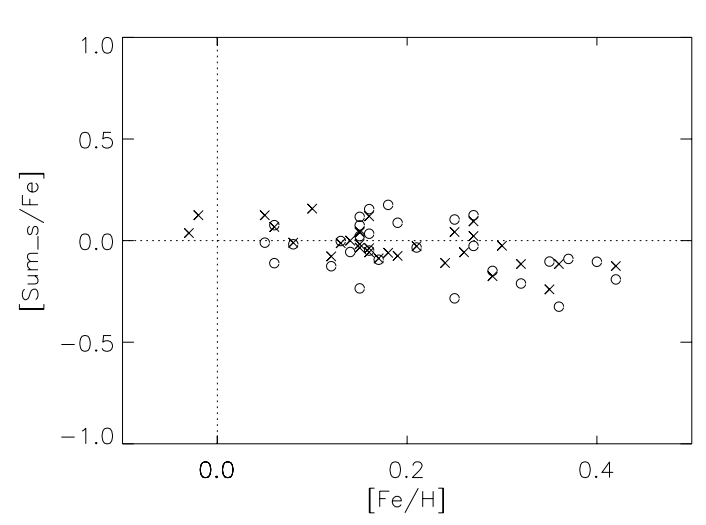

Fig. 24. The sums of light and heavy $s$-process elements are shown. The sums has been weighted as follows light $=0.5 \cdot[\mathrm{Y} / \mathrm{Fe}]+0.3 \cdot[\mathrm{Zr} / \mathrm{Fe}]+0.1 \cdot[\mathrm{Nd} / \mathrm{Fe}]$, heavy $=$ $0.25 \cdot[\mathrm{Mo} / \mathrm{Fe}]+0.5 \cdot[\mathrm{La} / \mathrm{Fe}]+0.25 \cdot[\mathrm{Hf} / \mathrm{Fe}]$. Only stars with at least two of the elements in the sum measured are shown. $\circ$ symbols denote the sum of the light $s$-process elements and $\times$ symbols denote the heavy elements 
of each element. The results are plotted vs. $[\mathrm{Fe} / \mathrm{H}]$ in Fig. 24, where the $\mathrm{K}$ dwarfs have been excluded. A downward slope of roughly the same magnitude for the "light" and the "heavy" elements is seen. This trend is in fact also present in most of the corresponding diagrams for the individual elements, although the scatter in larger, see Figs. 22 and 23. A slope of this magnitude can also be traced for the $s$-elements in Edvardsson et al. (1993a), Fig. 22.

A tendency of this type may, if true, indicate that $s$ elements enrichment occurs less frequently in metal-rich AGB stars. One may speculate that this might be because mass loss could finish their evolution earlier than for more metal-poor stars.

\subsection{Europium}

In some studies the iron abundance derived from Fe II lines are preferred as reference element for europium rather than abundances derived from Fe I lines. From our data this does not seem to be an obvious choice. Particularly, this is not so for the $\mathrm{K}$ dwarf stars, since europium with its low ionization energy, $5.7 \mathrm{eV}$, will remain highly ionized for all our stars irrespective of the effective temperature while almost all iron will be in the neutral state in the cool stars. Thus abundances derived from Fe II for these stars would be vulnerable to departures from LTE in the ionization equilibrium. In our study we derive iron abundances from, in general, more than 30 lines from Fe I and from four or three lines from Fe II. Thus, also statistically we could expect the atomic abundance to be better determined.

In Fig. 25 data from Woolf et al. (1995) and our data are plotted with iron abundances derived from Fe I and Fe II as reference, respectively. Europium shows a declining trend with metallicity from $[\mathrm{Fe} / \mathrm{H}]=-1.0$ to 0.0 dex and this trend now seems to continue unchanged for $[\mathrm{Fe} / \mathrm{H}]$ $>0.0$ dex.

Europium is well-correlated with oxygen as well as with the $\alpha$-elements, Fig. 26. This supports the idea that europium, oxygen and the $\alpha$-elements are all formed in the same type of events, supernovae type II.

It should finally be mentioned that the abundance trends (or lack of trends) discussed here are consistent with the results obtained for 9 solar-type dwarf stars in the interval $0.05 \operatorname{dex}<[\mathrm{Fe} / \mathrm{H}]<0.25$ dex by Tomkin et al. (1997). One exception from this, however, is $\mathrm{Eu}$ for which the latter results sooner suggest a slight increase in the $[\mathrm{Eu} / \mathrm{Fe}]$ with increasing $[\mathrm{Fe} / \mathrm{H}]$ on the basis of measurements of the same Eu lines as used here.

\section{Conclusions - summary}

We have determined elemental abundances for $47 \mathrm{G}$ and $\mathrm{K}$ dwarf stars with iron abundances ranging from solar
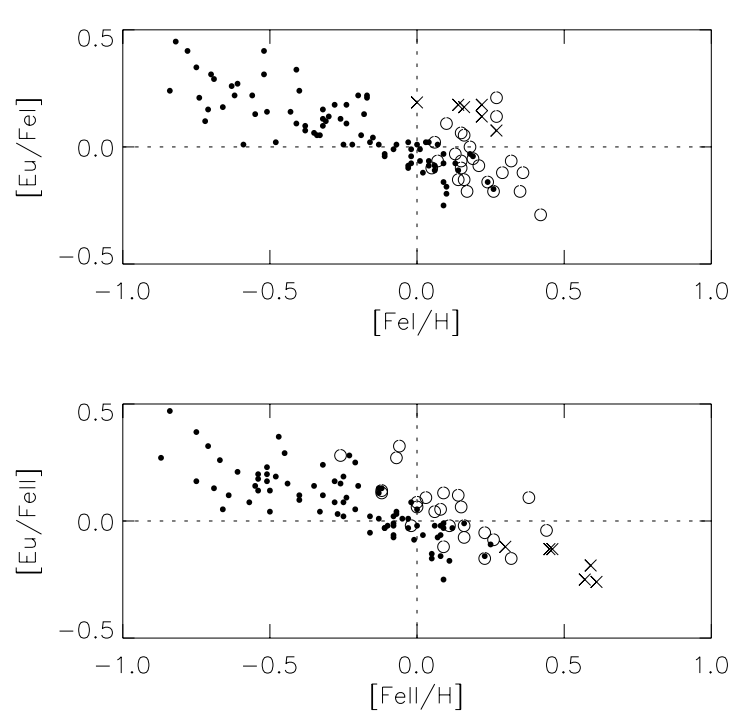

Fig. 25. Europium abundances. $\circ$ symbols this work, $\times$ symbols K dwarf stars this work, - symbols Woolf et al. (1995). Iron abundances are derived from FeI and Fe II, in the two panels respectively

to $[\mathrm{Fe} / \mathrm{H}]=0.4$ dex. The stars selected either move on relatively eccentric orbits spending most of their time inside the solar circle, even having been born there, or move on solar like orbits. Among the stars on solar like orbits there are also a number of Extreme Population I stars, with photometric logarithmic metallicities $[\mathrm{Me} / \mathrm{H}]>$ 0.4 dex. These samples were selected in an attempt to study differences in the chemical evolution in different parts of the galactic disk. Our main results and conclusions are summarized below.

The results fall roughly into two categories; galactic chemical evolution as studied from $\mathrm{G}$ dwarf stars and issues related to spectroscopy of dwarf stars and in particular to overionization in $\mathrm{K}$ dwarf stars.

\section{Galactic chemical evolution}

Our data do, in general, fit well into the overall picture of galactic chemical evolution as it is currently understood from empirical studies. They give further constraints on theoretical models of galactic chemical evolution for the region above solar metallicity, where metallicity dependent supernova yields further complicate the picture. In particular we find:

1. that $\mathrm{Mg}, \mathrm{Al}, \mathrm{Si}, \mathrm{Ti}, \mathrm{Ni}, \mathrm{Ca}$, and $\mathrm{Cr}$ follow iron also at these high metallicities,

2. that $[\mathrm{Fe} / \mathrm{H}]$ does not vary with $V_{\mathrm{LSR}}$, nor any other element. This indicates that the chemical evolution has lately been very homogeneous in the regions under study. This is in contrast with findings for $\alpha$-elements 


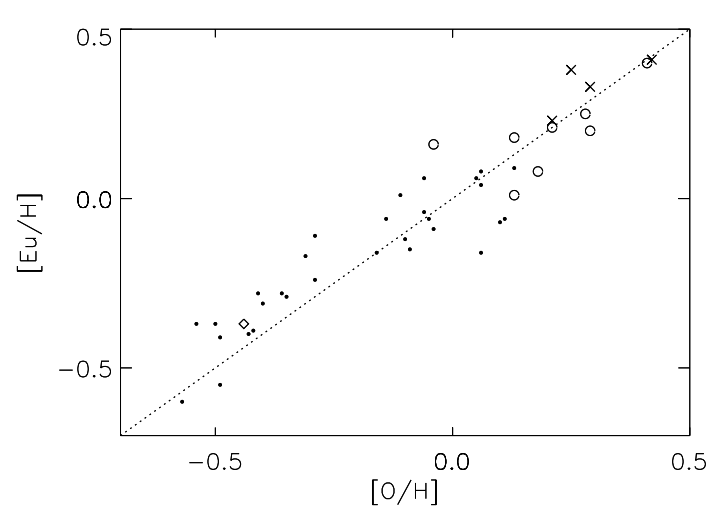

Fig. 26. Europium abundances and oxygen abundances compared. $\circ$ this work (excluding the $\mathrm{K}$ dwarf stars), $\times \mathrm{K}$ dwarf stars, $\bullet$ Woolf et al. (1995). The line with slope +1 is indicated by a dotted line. Oxygen abundances are in our work derived from the [O I] line at $6300 \AA$ while the oxygen abundances used together with the europium data from Woolf et al. (1995) are from Edvardsson et al. (1993a) and are derived (mostly) from the triplet lines at $7774 \AA$, scaled to the [O I] abundances using results from Nissen \& Edvardsson (1992)

in the more metal-poor part of the disk population, Edvardsson et al. (1993a),

3. that oxygen continues to decline with increasing $[\mathrm{Fe} / \mathrm{H}]$. This has also been confirmed by Castro et al. (1997),

4. that the upturn in $[\mathrm{Na} / \mathrm{Fe}]$ vs. $[\mathrm{Fe} / \mathrm{H}]$, as observed by Edvardsson et al. (1993a), is reproduced and reinforced. The origin of the upturn is, however, still somewhat unclear, but metallicity dependent supernova yields certainly play a role. In contrast to sodium we find no such upturn for aluminium. This is somewhat surprising since sodium and aluminium are thought to be produced in the same environment in the presupernova star.

Our lack of success in tracing variations in relative abundances, e.g. of $[\mathrm{O} / \mathrm{Fe}],[\mathrm{Na} / \mathrm{Fe}],[\mathrm{Si} / \mathrm{Fe}],[\mathrm{Ti} / \mathrm{Fe}]$ etc to vary with the stellar velocities, i.e. with the present orbital mean distance from the galactic centre $\left(R_{\mathrm{m}}\right)$. Thus, the probable mixture of the metal-rich stellar sample, e.g., of one old and one more recent population is not revealed as differences in relative abundances. That may be because of several reasons. One is that the relative number of stars from each population may not change very strongly with galactic radius. Another may be that the yields of heavy elements from the sites of nucleosyntheis are such that, e.g., the interstellar $\mathrm{Na} / \mathrm{Fe}$ ratio may stay rather constant through the evolution of the galactic disk. A third possibility is that galactic orbital diffusion is strong enough to bring these differences below the limit of detection. Thus, the observed scatter in such abundance ratios can not be explained as the result of the mixing of stars with differ- ent formation sites at different distances from the galactic centre.

For oxygen and europium our data suggest a continuation in the declining trend found by earlier studies at lower metallicities. This suggests that enrichment from supernovae Ia is still important, also at these relatively high metallicities.

The scatter in titanium abundances found in Edvardsson et al. (1993a) is reproduced in our data in spite of the fact that we use more lines to determine abundances from. It seems probable, but remains to be proven, that this scatter is "cosmic".

Stars with very high metallicities, so called Super Metal Rich, SMR, stars, have attracted some attention over the years. These stars have been claimed to either represent a late stage of the local galactic disk evolution or to be members of the Galactic bulge, which have now been scattered out to the solar neighborhood. Barbuy \& Grenon (1990) obtained photometric metallicities and oxygen abundances for eleven such stars. They found these stars to have high photometric metallicities and to be overabundant in oxygen. Castro et al. (1997) have obtained abundances for 9 similar stars. They find that oxygen, calcium and titanium relative to iron declines with increasing $[\mathrm{Fe} / \mathrm{H}]$, while sodium, silicon and nickel relative to iron increases. Several of these stars have fairly low temperatures and we interpret their results for calcium and nickel as further evidence for the phenomenon possibly arising from overionization. No velocity data have so far been published for these stars and therefore it is difficult to compare with our results. We have, however, obtained spectra and derived abundances for three of the stars in Barbuy \& Grenon (1990). These stars remain puzzling their proposed very high $[\mathrm{Fe} / \mathrm{H}]$ have not been verified, but at least some of them seem to have, as these authors suggest, a high $[\mathrm{O} / \mathrm{Fe}]$ ratio. This should be further explored.

\section{Overionization}

For the $\mathrm{K}$ dwarf stars we do detect what appears to be a case of overionization for, in particular, iron and chromium, such that abundances derived from Fe II and CrII are much higher that those derived from Fe I and Cr I. Fe I abundances do not vary with the effective temperature. Also for calcium and nickel the $\mathrm{K}$ dwarf stars depart markedly from the trend outlined by the G dwarf stars. Signs of similar departures for rare earth atoms are also traced.

Acknowledgements. Erik Hein Olsen is thanked for providing his catalogue prior to publication, invaluable for the planning of the project and subsequent analysis of the data. Poul-Erik Nissen is thanked for valuable comments and suggestions and 
for taking time to teach SF how to most efficiently reduce echelle spectra. Pierre Magain is thanked for compiling and sharing the individual line-to-line scatter for the studies from his and Zhao's work. Bengt Edvardsson is thanked for providing programs for the photometric calibration and comments during the work.

Karin Eriksson carried out a substantial fraction of all the measurements of equivalent widths and Matthias Palmer and Mikael Nilsson the determinations of surface gravities from the strong $6162 \AA$ Ca I line. We are much indebted to all three for these contributions.

The Swedish Royal Academy of Sciences is gratefully thanked for giving SF a grant to travel to the U.S.A. and thus being able to partake in the observational work at McDonald Observatory. This work was carried out while BG held the Beatrice Tinsley visiting professorship at the University of Texas, which is also gratefully acknowledged. The Observing Committee at McDonald Observatory generously granted observing time.

\section{References}

Abia C., Rebolo R., Beckman J.E., Crivallari L., 1988, A\&A 206, 100

Allen C.W., 1973, Astrophysical quantities. The Athlone Press

Andersen J., Gustafsson B., Lambert D.L., 1984, A\&A 136, 65

Anders E., Grevesse N., 1989, Geochim. Cosmochim. Acta 53, 197

Arribas S., Martinez Roger C., 1989, A\&A 215, 305

Asplund M., Gustafsson B, Kiselman D., Eriksson K., 1997, A\&A 318, 521

Aumann J.R., Woodrow J.E.J., 1975, ApJ 197, 163

Barbuy B., Grenon M., 1990, in Bulges of Galaxies, ESO/CTIO workshop, Jarvis B.J., Terndrup D.M. (eds.), p. 83

Baumueller D., Gehren T., 1996, A\&A 307, 961

Bell R.A., Gustafsson B., 1989, MNRAS 236, 653

Biémont E., Baudoux M., Kurucz R.L., Ansbacher W., Pinnington E.H., 1991, A\&A 249, 539

Blackwell D.E., Willis R.B., 1977, MNRAS 180, 169

Blackwell D.E., Lynas-Gray A.E., Smith G., 1995, A\&A 296, 217

Bruls J.H.M.J., 1993, A\&A 269, 509

Bruls J.H.M.J., Rutten R.J., Shchukina N.G., 1992, A\&A 265, 237

Carlsson M., Rutten R.J., Bruls J.H.M.J., Shchukina N.G., 1994, A\&A 288, 860

Cayrel de Strobel G., Bentolia C., 1983, A\&A 119, 1

Cayrel de Strobel G., Soubiran C., Friel E.D., Ralite N., Francois P., 1997, A\&AS 124, 299

Castro S., Rich R.M, Grenon M., Barbuy B., McCarthy J.K., 1997, AJ 114, 376

Delbouille L., Neven L., Roland G., 1973, Photometric atlas of the solar spectrum from $\lambda 3000$ to $\lambda 10000$

Drake J.J., 1991, MNRAS 251, 369

Edvardsson B., 1988, A\&A 190, 148

Edvardsson B., Andersen J., Gustafsson B., et al., 1993a, A\&A 275,101
Edvardsson B., Gustafsson B., Nissen P.E., et al., 1993b, in Panchromatic View of Galaxies, Hensler G., Theis Ch. \& Gallagher J. (eds.), p. 401

Eggen O.J., 1960, MNRAS 120, 430

Eriksson K., Toft S.C., 1979, A\&A 71, 178

Friel E., Cayrel de Strobel G., Chmielewski Y., et al., 1993, A\&A 274, 825

Gratton R.G., 1989, A\&A 208, 171

Gratton R.G., Sneden C., 1987, A\&A 178, 179

Gratton R.G., Sneden C., 1991, A\&A 241, 501

Grenon M, 1989, Astrophys. Space Sci. 156, 29

Griffin R.F., 1968, Photometric atlas of the spectrum of Arcturus, $\lambda \lambda 3600-8825 \AA$, Cambridge Philosophical Society

Gustafsson B., 1995, in Astrophysical Applications of Powerful New Databases, ASP Conf Ser. 78, Adelman S.J. \& Wiese W.L. (eds.), p. 347

Gustafsson B, Kjaergaard P., Andersen S., 1974, A\&A 34, 99

Gustafsson B., Bell R.A., Eriksson K., Nordlund Å., 1975, A\&A 42, 407

Hannaford P., Lowe R.M., Grevesse N., Noels A., 1992, A\&A 259, 301

Hoffleit D., Jascheck C., 1982, The Bright Star Catalogue, Yale University Observatory

Holweger H., 1971, A\&A 10, 128

Holweger H., Bard A., Kock A., Kock M., 1991, A\&A 249, 545

Johnson H.L., 1966, ARA\&A 4, 193

Kiselman D., 1993, Thesis (Uppsala University) and A\&A 275, 269

Kiselman D., Nordlund Å., 1995, A\&A 302, 578

Kurucz R.L., 1989, Magnetic tapes with atomic line data for Ca through Ni (private communication)

Kurucz R., Furenlid I., Brault J., Testerman L., 1984, Solar Flux Atlas from 296 to 1300 nm, National Solar Observatory, Sunspot, New Mexico

Lambert D.D., 1978, MNRAS 182, 249

Mäckle R., Holweger H., Griffin R., Griffin R., 1975, A\&A 38, 239

Mathews G.J., Bazan G., Cowan J.J., 1992, ApJ 391, 719

Matteucci F., François P., 1989, MNRAS 239, 885

McWilliam A., 1990, ApJS 74, 1075

Moore C.E., Minnaert M.J.G., Houtgast J., 1966, The solar spectrum $2935 \AA$ to $8770 \AA$, National Bureau of Standards Monograph 61

Morell O., 1994, PhD Thesis, Acta Universitas Upsaliensis ISBN 91-554-3322-7

Neff J.E., O’Neal D., Saar S.H., 1995, ApJ 452, 879

Neuforge C., 1992, in Origin and Evolution of the Elements, Prantzos N., Vangioni-Flam E., Cassé M. (eds.), p. 63

Nissen P.E., Edvardsson B., 1992, A\&A 261, 255

Nissen P.E., Gustafsson G., Edvardsson B., Gilmore G., 1994, A\&A 285, 440

Olsen E.H., 1983, A\&AS 54, 55

Olsen E.H., 1984, A\&AS 57, 443

Olsen E.H., 1993, A\&AS 102, 89

Olsen E.H., 1994, A\&AS 106, 257

Pagel B.E.J., Tautvaišienè G., 1995, MNRAS 276, 505

Prantzos N., Aubert O., 1995, A\&A 302, 69

Ruland F., Holweger H., Griffin R., Griffin R., Biehl D., 1980, A\&A 92, 70 
Ruland F., Griffin R., Griffin R., Biehl D., Holweger H., 1981, A\&AS 42, 391

Rutten R.J., 1988, in Physics of Formation of FeII Lines Outside LTE, Viotti R., Vittone A., Friedjung M. (eds.), IAU Coll. 94. Reidel Publ. Company, p. 185

Schuster W.J., Nissen P.E., 1988, A\&AS 73, 225

Smith G., 1981, A\&A 103, 351

Steenbock W., 1985, in Cool stars with Excess of Heavy Elements, Jaschek M. \& Keenan P.C. (eds.). D. Reidel Publ. Company, p. 231

Steenbock W., Holweger H., 1984, A\&A 130, 319

Summers K., 1994, Undergraduate thesis at the Astronomical Observatory, Uppsala University

Taylor B.J., 1996, ApJS 102, 105

Thielemann K-F., Nomoto K., Hashimoto M., 1996, ApJ 460, 408

Timmes F.X., Woosley S.E., Weaver T.A., 1995, ApJS 98, 617
Tomkin J., Woolf V.M., Lambert D.L., Lemke M., 1995, AJ 109,2204

Tomkin J., Edvardsson B., Lambert D.L., Gustafsson B., 1997, A\&A (in press)

Tsujimoto T., Yoshii Y., Nomoto K., Shigeyama T., 1995, A\&A 302,704

Tull T.G., MacQueen P.J., Sneden C., Lambert D.L., 1995, PASP 107, 251

VandenBerg D.A., 1992, ApJ 391, 685

van Altena W.F, Truen-Liang Lee J., Hoffleit D., 1991, Yale Trigonometric Parallaxes Preliminary. Yale, University Observatory

Wielen R., Fuchs B., Dettbarn C., 1996, A\&A 314, 438

Woolf V.M., Tomkin J., Lambert D.L., 1995, ApJ 453, 660

Woosley S.E., Weaver T.A., 1995, ApJS 101, 181

Wyse R., Gilmore G., 1995, AJ 110, 2771

Zhao G., Magain P., 1990, A\&A 238, 242 